\title{
On supersymmetric D7-branes in the warped deformed conifold
}

\author{
Heng-Yu Chen, ${ }^{a}$ Peter Ouyang ${ }^{a}$ and Gary Shiu ${ }^{a, b}$ \\ ${ }^{a}$ Department of Physics, University of Wisconsin, \\ Madison, WI 53706, U.S.A. \\ ${ }^{b} \mathrm{PH}-\mathrm{TH}$ Division, CERN, \\ CH-1211 Geneva 23, Switzerland \\ E-mail: hchen46@wisc.edu, pouyang@wisc.edu, shiu@physics.wisc.edu
}

ABSTRACT: We study the supersymmetric properties of D7-branes in the warped deformed conifold. We consider the $\kappa$-symmetry conditions on D7-branes in this specific warped background, taking into account the background NS-NS 2-form flux. While any holomorphic embedding defines a supersymmetric D7-brane in the absence of background H-flux, most of the D7-brane embeddings considered in the literature do not preserve supersymmetry for the warped deformed conifold without also including brane worldvolume flux. For the simplest such embedding, we construct numerically the worldvolume flux necessary to restore supersymmetry. We also comment on the dual field theory descriptions in terms of cascading $\mathcal{N}=1$ supersymmetric gauge theories with flavors. Finally, we discuss some possible applications of our results to moduli stabilization and vacuum energy uplifting, gauge/gravity duality, and string inflationary model building.

KEYWORDS: Gauge-gravity correspondence, D-branes

ARXIV EPRINT: 0807.2428 


\section{Contents}

1 Introduction 1

2 Review of the warped deformed conifold 3

$\begin{array}{lll}3 & \text { Supersymmetry conditions } & 7\end{array}$

4 Supersymmetric D7 embeddings in the conifold 8

$4.1 z$-embedding 9

$\begin{array}{lll}4.2 & w \text {-Embedding } & 10\end{array}$

$\begin{array}{lll}4.3 & \text { Restoring supersymmetry with worldvolume flux } & 11\end{array}$

$\begin{array}{lll}\text { 4.3.1 } & \text { Boundary conditions and finiteness } & 14\end{array}$

$\begin{array}{ll}\text { 4.3.2 Large radius "factorization" limit } & 15\end{array}$

4.3.3 Large radius "interpolating" limit 16

$\begin{array}{lll}\text { 4.3.4 Consistent boundary conditions and subleading asymptotics } & 18\end{array}$

$\begin{array}{ll}\text { 4.3.5 Numerical solution } & 20\end{array}$

$\begin{array}{ll}\text { 4.3.6 Summary of results } & 21\end{array}$

$\begin{array}{lll}4.4 & \text { Other embeddings } & 23\end{array}$

5 Field theory remarks $\quad \mathbf{2 4}$

5.1 Cascade pattern for $z$ embedding and its generalizations 25

$\begin{array}{ll}5.2 & \text { The vacua for Kuperstein-like embeddings }\end{array}$

$5.3 w$-embedding and its generalizations 28

6 Discussion $\quad 29$

$\begin{array}{ll}\text { A Deformed conifold coordinates } & 31\end{array}$

B Generalized $z$-embeddings $\quad 31$

C Karch-Katz embedding 33

$\begin{array}{ll}\text { D Comparison with Benini's proposal } & 34\end{array}$

\section{Introduction}

One of the major developments in string theory of the last decade has been the emergence of warped spacetimes as a setting for a rich variety of physics. To date, perhaps the most important application of such warped geometries has been in the gauge theory/string theory correspondence [1-3]. In this setting, noncompact spacetimes which are warped 
by the presence of background flux have been conjectured to give a dual description of gauge theories. In many cases, this duality can relate strongly coupled field theories to weakly coupled gravity theories, and as such offers new tools for studying field theories when traditional perturbative methods fail.

Another application of warped geometry has been in the study of string theory as a theory of particle physics. When one compactifies string theory from ten dimensions to four dimensions, the compactification manifold can support nontrivial background fluxes, and these fluxes will generically have a backreaction on the geometry, resulting in warping. The gravitational redshift generated by a strong warp factor can lead to a hierarchy of scales, thus offers a geometrical explanation for the huge disparity between the electroweak and the Planck scale [4, 5] (see also [6-9] for string theory realizations). For the same reason, warped geometries have also been invoked in supersymmetry breaking scenarios in string theory, both to lower the scale of supersymmetry breaking [10, 11] and to control the amount of sequestering [12]. The low energy effective supergravity theory describing strongly warped backgrounds is challenging to derive, though continued progress has been made [10, 11, 13-17].

Warping has also found application in inflationary cosmology. A key issue in inflationary cosmology is to understand the underlying dynamics which allows for a sufficient number e-folds of expansion. It turns out that warping can be fruitfully used in stringy cosmological models to construct suitable potentials for slow roll inflation as well as for motivating new inflationary mechanisms (see [18-23] and the references therein).

Strikingly, there is a particular warped geometry, the warped deformed conifold in type IIB supergravity [24, 25], which is simple enough to allow detailed study, but is also rich enough to have been used in all three of these areas. It has almost anti-de Sitter asymptotics, and in gauge/gravity duality it is dual to an interesting confining gauge theory. If one embeds the deformed conifold in a compact Calabi-Yau, and turns on appropriate three-form fluxes, one finds that the resulting background has a natural and stable hierarchy of scales [9]. And in cosmology, interesting inflationary models have been also constructed in the conifold [26-29].

In this paper, we study a particularly interesting variation of the warped deformed conifold, in which we add a certain number of probe D7-branes to the background. These branes fill four noncompact directions and wrap a four-cycle $\Sigma_{4}$ in the transverse space. In the gauge theory dual, these branes add fundamental matter to the theory [30, 31], offering the prospect of field theory duals which resemble QCD. Moreover, in more phenomenological applications, the D7-branes allow the introduction of gaugino condensates, which can stabilize Kähler moduli [32] and which can introduce potentials for mobile D3-branes (candidate inflatons in the scenarios considered in [26-28]). Given this range of applications, it is of clear interest to find as many supersymmetric D7-brane embeddings in the deformed conifold as possible, allowing for greater freedom in model-building. In this paper we study the supersymmetry conditions for several different classes of branes, focusing in particular on the cases where worldvolume flux is necessary for the brane to preserve supersymmetry.

This paper is organized as follows. We begin by reviewing the deformed conifold in section 2, along the way collecting some results which will be useful in the rest of the 
paper. In section 3, we review the criteria for the D7-branes to preserve supersymmetry (the $\kappa$-symmetry conditions) in a general Calabi-Yau flux compactification. We make use of these results in section 4, where we study the case of D7-branes in the conifold with background fluxes. There is one such D7-brane embedding (sometimes known as the Kuperstein embedding) already known to be supersymmetric with no worldvolume flux turned on [33]. On the other hand, the embedding first studied in [34] is not supersymmetric when the worldvolume flux vanishes. Our main result is in section 4.3, where we construct the worldvolume flux necessary to restore supersymmetry, by a combination of analytic and numerical methods. We also offer a criterion that in many cases can show whether a worldvolume flux is needed to restore supersymmetry, and which is far simpler to verify than explicitly checking $\kappa$-symmetry. In section 5 , we make some observations about the field theories dual to the conifold with our D7-branes included. Finally, we end with a discussion of prospects for the future in section 6 .

\section{Review of the warped deformed conifold}

In this paper we focus on a particular concrete example of a warped Calabi-Yau space, namely the warped deformed conifold. This geometry is noncompact, but in principle it can be embedded in a compact Calabi-Yau with background bulk fluxes. We now proceed to review some facts about the conifold and warped compactifications in general which will be useful in the rest of the paper.

The conifold is most simply defined as a submanifold of flat $\mathbb{C}^{4}$ given by the equation $[24]$

$$
z_{1}^{2}+z_{2}^{2}+z_{3}^{2}+z_{4}^{2}=0
$$

where the $z_{i}$ are coordinates of the ambient $\mathbb{C}^{4}$. This space has a manifest $\mathrm{SU}(2) \times \mathrm{SU}(2) \times$ $\mathrm{U}(1)$ global symmetry, where the $\mathrm{SU}(2) \times \mathrm{SU}(2) \simeq \mathrm{SO}(4)$ acts by rotating the $z_{i}$ and the $\mathrm{U}(1)_{R}$ acts as multiplication of each of the $z_{i}$ by a phase, $z_{i} \rightarrow e^{i \varphi} z_{i}$. The defining equation of the conifold is also invariant under an overall rescaling of the coordinates, which implies that it admits a conical metric. The corresponding geometry is a cone over a five-dimensional Einstein manifold called $T^{1,1}$ and the metric takes the form

$$
d s_{6}^{2}=d r^{2}+r^{2} d s_{T^{1,1}}^{2} .
$$

where the metric of the $T^{1,1}$ base is

$$
d s_{T^{1,1}}^{2}=\frac{1}{9}\left(d \psi+\sum_{i=1}^{2} \cos \theta_{i} d \phi_{i}\right)^{2}+\frac{1}{6} \sum_{i=1}^{2}\left(d \theta_{i}^{2}+\sin ^{2} \theta_{i} d \phi_{i}^{2}\right)
$$

and the angular coordinates range as $0 \leq \psi \leq 4 \pi, 0 \leq \theta_{i} \leq \pi$, and $0 \leq \phi_{i} \leq 2 \pi$. From the form of the metric it is clear that base is a sphere fibration $S^{1} \times S^{2} \times S^{2}$ and it turns out that its topology is actually $S^{3} \times S^{2}$. The base space has Betti numbers $b_{2}=b_{3}=1$, and the associated harmonic forms are

$$
\begin{aligned}
& \omega_{2}=\frac{1}{2}\left(\Omega_{11}-\Omega_{22}\right) \\
& \omega_{3}=\zeta \wedge \omega_{2}
\end{aligned}
$$


where $\zeta=d \psi+\cos \theta_{1} d \phi_{1}+\cos \theta_{2} d \phi_{2}$ and

$$
\Omega_{i j}=d \theta_{i} \wedge \sin \theta_{j} d \phi_{j}
$$

This space is singular at the tip of the cone where all the $z_{i}$ are zero (and so we will sometimes refer to this space as the "singular conifold.")

It is also possible to define the conifold in terms of another set of complex coordinates, by the equation

$$
w_{1} w_{2}-w_{3} w_{4}=0
$$

which is related to the original defining equation by an obvious change of variables. It turns out that this alternative description will also be useful to us. One nice property is that the $w_{i}$ coordinates admit a relatively simple description in terms of the angles on $T^{1,1}$ :

$$
\begin{aligned}
& w_{1}=r^{3 / 2} e^{i / 2\left(\psi-\phi_{1}-\phi_{2}\right)} \sin \frac{\theta_{1}}{2} \sin \frac{\theta_{2}}{2}, \\
& w_{2}=r^{3 / 2} e^{i / 2\left(\psi+\phi_{1}+\phi_{2}\right)} \cos \frac{\theta_{1}}{2} \cos \frac{\theta_{2}}{2}, \\
& w_{3}=r^{3 / 2} e^{i / 2\left(\psi-\phi_{1}+\phi_{2}\right)} \sin \frac{\theta_{1}}{2} \cos \frac{\theta_{2}}{2}, \\
& w_{4}=r^{3 / 2} e^{i / 2\left(\psi+\phi_{1}-\phi_{2}\right)} \cos \frac{\theta_{1}}{2} \sin \frac{\theta_{2}}{2} .
\end{aligned}
$$

One of the important properties of the conifold is that its singularity can be smoothed by adjusting moduli. At the tip of the cone, one may perform either a small resolution, blowing up an $S^{2}$, or a deformation, by changing the defining equation of the conifold to

$$
z_{1}^{2}+z_{2}^{2}+z_{3}^{2}+z_{4}^{2}=\epsilon^{2} .
$$

We can make a phase rotation of the $z_{i}$ so that $\epsilon$ is real, and we will always assume this in the rest of the paper. It is evident from taking a real slice of this defining equation that deformation results in the appearance of a finite-sized $S^{3}$ at the tip of the cone. The deformed conifold preserves the $\mathrm{SO}(4)$ isometry of the singular conifold, but the scale invariance is broken by $\epsilon$ and the $\mathrm{U}(1)_{R}$ symmetry is broken to a $\mathbb{Z}_{2}$ subgroup.

This deformed geometry has special relevance in flux compactifications because of its topology. The existence of a three-cycle allows us to turn on RR 3-form flux on this cycle, and there are supersymmetric supergravity solutions if we also include NS-NS flux on the (noncompact) dual cycle.

We now proceed to review flux compactification of the type considered in [9], where a warped deformed conifold throat naturally develops near the local conifold singularities. Let us begin with the ten dimensional warped metric preserving four-dimensional Poincare symmetry which takes the following form

$$
d s_{10}^{2}=e^{2 A(y)} \tilde{g}_{\mu \nu} d x^{\mu} d x^{\nu}+e^{-2 A(y)} \tilde{g}_{m n} d y^{m} d y^{n} .
$$

Here $\tilde{g}_{\mu \nu}, \mu, \nu=0,1,2,3$ and $\tilde{g}_{m n}, m, n=4, \ldots, 9$ are the unwarped metrics for the non-compact four dimensional space time and the compact six dimensional manifold $\mathcal{M}_{6}$ respectively, whereas $e^{A(y)}$ is the warp factor which only varies over $\mathcal{M}_{6}$. 
Following [9], we shall allow for both RR and NS-NS fluxes of IIB supergravity to be turned on, and they can be written succinctly as:

$$
\begin{aligned}
& G_{3}=F_{3}-\tau H_{3}=\frac{1}{6} G_{m n p}\left[d y^{m} \wedge d y^{n} \wedge d y^{p}\right], \\
& \tilde{F}_{5}=F_{5}-\frac{1}{2} C_{2} \wedge H_{3}+\frac{1}{2} B_{2} \wedge F_{3}=\left(1+*_{10}\right)\left[d \alpha(y) \wedge d x^{0} \wedge d x^{1} \wedge d x^{2} \wedge d x^{3}\right] .
\end{aligned}
$$

Here we have allowed for IIB axio-dilaton $\tau \equiv \tau(y)=C_{0}-i e^{-\phi}$ to vary over $\mathcal{M}_{6}$ and in $(2.15)$ we have used self-duality $\tilde{F}_{5}=*_{10} \tilde{F}_{5}$, four dimensional Poincare invariance and Bianchi identity to constrain the expression for $\tilde{F}_{5}$. In addition, local objects extending into non-compact four dimensions can also be included (which may wrap cycles in $\mathcal{M}_{6}$ ), and they need to satisfy the tadpole cancellation condition:

$$
\frac{1}{2 \kappa_{10}^{2} T_{3}} \int_{\mathcal{M}_{6}} H_{3} \wedge F_{3}+Q_{3}^{\text {local }}=0
$$

where $Q_{3}^{\text {local }}$ is the D3-brane charge on the local objects. The supergravity equation of motion for such configuration of fluxes and local sources then yields:

$$
\tilde{\nabla}^{2} \Phi_{ \pm}=\frac{e^{A}}{6 \operatorname{Im}(\tau)}\left|G_{ \pm}\right|^{2}+\left|\nabla \Phi_{ \pm}\right|^{2}+2 \kappa_{10}^{2} e^{2 A}\left[\frac{\left(T_{m}^{m}-T_{\mu}^{\mu}\right)^{\text {local }}}{4}-T_{3} \rho_{3}^{\text {local }}\right]
$$

where we have defined the following linear combination:

$$
\Phi_{ \pm}=e^{4 A(y)} \pm \alpha(y), \quad G_{ \pm}=i G_{3} \pm *_{6} G_{3},
$$

and $T_{\mu \nu}$ and $T_{m n}$ are four and six dimensional energy-momentum tensors. A special solution to (2.17) is given by the so-called imaginary self-dual (ISD) configuration:

$$
\begin{gathered}
\Phi_{-}=0, \quad G_{-}=0, \quad \leftrightarrow \quad e^{4 A(y)}=\alpha(y), \quad *_{6} G_{3}=i G_{3}, \\
\frac{\left(T_{m}^{m}-T_{\mu}^{\mu}\right)^{\text {local }}}{4}=T_{3} \rho_{3}^{\text {local }} .
\end{gathered}
$$

In terms of the local sources in IIB, the equality (2.20) can be satisfied by D3 branes or O3 planes, D5 brane wraps on vanishing two cycle, also known as "fractional D3"; or D7 wraps on non-trivial four cycles, e.g. K3. Turning now from these generalities back to the conifold, let us turn on $M$ units of $F_{3}$ along the so-called A-cycle which corresponds to the minimal $S^{3}$ in the deformed conifold, and also $(-K)$ units of $H_{3}$ in the dual B-cycle. The quantization condition for the fluxes then yields:

$$
\frac{1}{2 \pi \alpha^{\prime}} \int_{A} F_{3}=M, \quad \frac{1}{2 \pi \alpha^{\prime}} \int_{B} H_{3}=-K
$$

These three form fluxes stabilize the value of $\epsilon^{2}$ at

$$
\epsilon^{2} \sim \exp \left(-2 \pi K / g_{s} M\right),
$$

and the warp factor at the tip is given by $e^{A_{0}}=e^{-2 \pi K / 3 M g_{s}}$. The total D3 charge is given by the intersection form:

$$
\frac{1}{2 \kappa_{10}^{2} T_{3}} \int_{\mathcal{M}_{6}} H_{3} \wedge F_{3}=M K=N .
$$


The explicit IIB supergravity solution corresponds to the flux configuration described here was given in [25], where the authors started with $N$ unit of D3 branes and $M$ unit of fractional D3 branes at the tip of singular conifold, with $g_{s} N, g_{s} M \gg 1$. In the near horizon limit, these branes are replaced by their fluxes, one can obtain explicit expressions for the corresponding warp factor and fluxes (for reviews, see e.g. [35, 36]).

In the large radius limit, the supergravity solution including these 3 -form fluxes (but with the axion and dilaton constant) has the useful description (due to Klebanov and Tseytlin, or KT) [37]:

$$
\begin{aligned}
d s_{10}^{2} & =e^{2 A(r)}\left(d x_{\mu} d x^{\mu}\right)+e^{-2 A(r)}\left(d r^{2}+r^{2} d s_{T^{1,1}}^{2}\right) \\
e^{-4 A(y)} & =\frac{27}{4 r^{4}} \pi g_{s} \alpha^{\prime 2}\left(N+\frac{3}{2 \pi} g_{s} M^{2} \log \left(r / r_{0}\right)\right) \\
g_{s} \tilde{F}_{5} & =d^{4} x \wedge d e^{4 A(r)}+*_{10}\left(d^{4} x \wedge d e^{4 A(r)}\right) \\
F_{3} & =\frac{M \alpha^{\prime}}{2} \omega_{3} \\
B_{2} & =\frac{3 g_{s} M \alpha^{\prime}}{2} \log \left(r / r_{0}\right) \wedge \omega_{2} \\
H_{3} & =d B_{2}=\frac{3 g_{s} M \alpha^{\prime}}{2} \frac{d r}{r} \wedge \omega_{2} .
\end{aligned}
$$

This solution possesses a naked singularity at small $r$; however, in the full KS solution the deformation of the conifold resolves the singularity.

For later use, we will record here the explicit Kähler form and the NS-NS B-field on the warped deformed conifold. $\mathrm{SO}(4)$ invariant expressions can be found in [35]:

$$
\begin{aligned}
J & =\mathcal{K}^{\prime}(\rho)\left(\sum_{i=1}^{4} d z_{i} \wedge d \bar{z}_{i}\right)+\mathcal{K}^{\prime \prime}(\rho)\left(\sum_{i=1}^{4} \bar{z}_{i} d z_{i}\right) \wedge\left(\sum_{j=1}^{4} z_{j} d \bar{z}_{j}\right), \\
B_{2} & =b(\rho) \epsilon_{i j k l} z_{i} \bar{z}_{j} d z_{k} \wedge d \bar{z}_{l}, \quad b(\rho)=\frac{i g_{s} M \alpha^{\prime}}{2|\epsilon|^{4}} \frac{\rho \operatorname{coth} \rho-1}{\sinh ^{2} \rho},
\end{aligned}
$$

where $\mathcal{K}(\rho)$ the Kähler potential for the deformed conifold is defined implicitly as:

$$
\begin{aligned}
\mathcal{K}^{\prime}(\rho) & =\left(\frac{\sinh (2 \rho)-2 \rho}{2|\epsilon|^{2} \sinh ^{3} \rho}\right)^{\frac{1}{3}}, \\
\mathcal{K}^{\prime \prime}(\rho) & =\frac{(5+\cosh (2 \rho)-6 \rho \operatorname{coth} \rho)}{6|\epsilon|^{2} \sinh \rho(\rho-\cosh \rho \sinh \rho)} \mathcal{K}^{\prime}(\rho) .
\end{aligned}
$$

Here ()$^{\prime}$ denotes the differentiation with respect to $r^{3}$, and the usual radial coordinate $r$ is related to the coordinate $\rho$ by

$$
r^{3}=\sum_{i=1}^{4}\left|z_{i}\right|^{2}=|\epsilon|^{2} \cosh \rho .
$$

The following definitions, taken from [36], prove to be notationally convenient:

$$
\eta_{1}=\epsilon_{i j k l} z_{i} \bar{z}_{j} d z_{k} \wedge d \bar{z}_{l}, \quad \eta_{4}=\sum_{i=1}^{4} \bar{z}_{i} d z_{i} \wedge \sum_{j=1}^{4} z_{j} d \bar{z}_{j}, \quad \eta_{5}=\sum_{i=1}^{4} d z_{i} \wedge d \bar{z}_{i}
$$


and we will make use of these forms in the calculations to follow. In the large $\rho$ limit, $b(\rho) \sim$ $\rho \epsilon^{-4} \exp (-2 \rho) \sim r^{-6} \log (r)$ and one finds that the potential $B_{2}$ varies logarithmically with $r$, as in [37].

\section{Supersymmetry conditions}

Given the rich physics that has arisen from the study of D7-branes in warped flux compactifications, it is interesting to find as many different stable configurations of D7-branes in these backgrounds as is possible. Because supersymmetry guarantees stability, and is typically a simpler property to check than non-supersymmetric stability, we will focus on a search for supersymmetric D7-branes in warped throats with flux. We begin by reviewing the supersymmetric embedding conditions given in [38, 39] for D7 branes wrapping a four-cycle in a generic Calabi-Yau three-fold, and then in the next section we will apply these conditions to specific examples of embeddings in the warped deformed conifold.

Consider a CY three-fold $\mathcal{M}_{6}$ with Kähler two-form $J$, a background NS-NS 2-form potential $B_{2}$, and some number of coincident D7-branes which fill out the four transverse Minkowski space-time dimensions and extend in four directions on the compact manifold. There is a vector field $A$ supported on the D7-brane worldvolume, and its curvature $F_{2}=d A$ combines with the background $B_{2}$ to form a gauge invariant combination $\hat{\mathcal{F}}$.

$$
\hat{\mathcal{F}}=\hat{B}_{2}+2 \pi \alpha^{\prime} F_{2},
$$

where we use ^ to denote the pull-back of bulk quantities on to the D7-brane worldvolume.

In $[38,39]$, it was found that the $\kappa$-symmetry condition for D7 branes wrapping a four-cycle $\Sigma_{4}$ in $\mathcal{M}_{6}$ are as follows:

1. The four cycle $\Sigma_{4}$ must be holomorphic. In other words, the D7-brane locus may be written as a holomorphic equation in the complex structure of the CY.

2. The generalized $\hat{\mathcal{F}}$ field strength is a two-form of pure $(1,1)$ type, or

$$
\hat{\mathcal{F}}^{2,0}=\hat{\mathcal{F}}^{0,2}=0 .
$$

3. The Kähler form $J$ pulled back to $\Sigma_{4}$ and the generalized field strength $\mathcal{F}$ satisfy the equation:

$$
e^{-A} \hat{J} \wedge \hat{\mathcal{F}}=\tan \theta\left(\frac{e^{2 A}}{2} \hat{J} \wedge \hat{J}-\frac{1}{2} \hat{\mathcal{F}} \wedge \hat{\mathcal{F}}\right)
$$

In (3.3) we have included the warp factor $e^{A}$ in (2.13) and the constant $\theta$ is specified by the supersymmetry conditions for a given supergravity background solution [38]. These conditions are equivalent to the requirement that the D7-brane configuration is a local energy minimum, and in the fluxless case are equivalent to the condition that $\Sigma_{4}$ is a minimal surface.

$\hat{\mathcal{F}}$ must also satisfy a modified Bianchi identity:

$$
d \hat{\mathcal{F}}=\hat{H}_{3},
$$


where $\hat{H}_{3}$ is the pull-back of $H_{3}=d B_{2}$. Equation (3.4) arises from the definition of $\hat{\mathcal{F}}$ when the gauge field strength by itself is closed and is necessary to guarantee cancellation of the global anomaly for open fundamental strings ending on the D7 branes [40]. Taken together, the condition (3.4) and the supersymmetry conditions are sufficient conditions for the equations of motion from the D7 brane action to be satisfied.

In the case of the warped deformed conifold, the parameter $\theta$ is zero and the condition (3.3) simplifies to

$$
\hat{J} \wedge \hat{\mathcal{F}}=0
$$

$\hat{\mathcal{F}}$ satisfying (3.5) is said to be "primitive." An alternative and very useful interpretation [38] for conditions (3.2) and (3.5) is that the generalized field strength $\hat{\mathcal{F}}$ is anti-selfdual:

$$
\hat{\mathcal{F}}=-*_{4} \hat{\mathcal{F}}
$$

where the Hodge star operation $*_{4}$ is taken with respect to the induced metric on the four cycle.

In terms of complex embedding coordinates $\left\{z_{i}\right\}, J$ and $B_{2}$ are manifestly $(1,1)$. Therefore in the absence of the magnetic field $F_{2}, \mathcal{F}$ is also of the type $(1,1)$ satisfying the supersymmetry condition eq. (3.2).

\section{Supersymmetric D7 embeddings in the conifold}

In this section we shall examine several holomorphic D7-brane embeddings in the singular conifold that have appeared in the literature. In the absence of background H-flux, the only requirement for a D7-brane to be supersymmetric is holomorphy of its embedding equation. Several such holomorphic embeddings have been proposed, and in the singular conifold limit, the $\kappa$-symmetry conditions are trivially satisfied, and in some cases the supercharges have been explicitly constructed [43]. However, the supersymmetry conditions are more complicated when H-flux is turned on, and our goal in this section is to explore this situation in detail.

We will focus first on linear holomorphic embeddings in the interest of simplicity. There are two natural classes of linear embeddings, given by either the description of the conifold in terms of the $z_{i}$ coordinates or the $w_{i}$ coordinates. The embedding $z_{1}=\mu$ was studied in detail in [33] and it satisfies the supersymmetry conditions (3.5) even on the deformed conifold without needing any additional world volume flux. Curiously, among the commonly studied classes of embeddings it appears to be the only one that does not require a flux.

Another simple class of linear embeddings was introduced in [34] and is most naturally expressed in the $w_{i}$ coordinates as, for example, $w_{1}=\mu$. When nontrivial $\mathrm{H}$-flux is turned on, this embedding is not supersymmetric without also turning on the world volume field strength $F_{2}$. We will exhibit the necessary flux, thus demonstrating that this class of D7-branes can in fact be made supersymmetric. To make the calculations tractable we have taken the large-radius (KT) limit of the warped deformed conifold, but it would be interesting to study these branes in the full KS geometry as well. 
In the following section we will refer to the relevant D7-branes as being in either the " $z$ embedding" or the " $w$-embedding", depending on the natural coordinates of the embedding equation. Having understood the simplest D7-brane embeddings, we will also see that many other more complicated embeddings will require worldvolume flux to be supersymmetric.

\section{$4.1 \quad z$-embedding}

We begin by considering the embedding for the deformed conifold proposed in [33]

$$
z_{4}=\mu, \quad \mu \in \mathbb{C} .
$$

This embedding breaks the $\mathrm{SO}(4)$ isometry group of the deformed conifold down to the $\mathrm{SO}(3)$ which rotates $\left\{z_{1}, z_{2}, z_{3}\right\}$. It was shown to be supersymmetric in the deformed conifold for real $\mu$ [33]. We review the calculation here, generalizing as well to complex $\mu$.

In order to check the supersymmetric condition (3.3), the pull back of the NS-NS B-field for this embedding can be obtained from

$$
\hat{\eta}_{1}=\left.\eta_{1}\right|_{z_{4}=\mu}=B_{1 \overline{1}} d z_{1} \wedge d \bar{z}_{1}+B_{1 \overline{2}} d z_{1} \wedge d \bar{z}_{2}+B_{2 \overline{1}} d z_{2} \wedge d \bar{z}_{1}+B_{2 \overline{2}} d z_{2} \wedge d \bar{z}_{2},
$$

where the various components are given by:

$$
\begin{aligned}
& B_{1 \overline{1}}=-\left|z_{3}\right|^{-2}\left(z_{1} \bar{z}_{3}-z_{3} \bar{z}_{1}\right)\left(z_{2} \bar{\mu}-\bar{z}_{2} \mu\right) \\
& B_{1 \overline{2}}=\left(z_{3} \bar{\mu}-\bar{z}_{3} \mu\right)+\left|z_{3}\right|^{-2}\left(z_{1} \bar{z}_{3}\left(z_{1} \bar{\mu}-\bar{z}_{1} \mu\right)+\bar{z}_{2} z_{3}\left(z_{2} \bar{\mu}-\bar{z}_{2} \mu\right)\right) \\
& B_{2 \overline{1}}=-\left(z_{3} \bar{\mu}-\bar{z}_{3} \mu\right)-\left|z_{3}\right|^{-2}\left(\bar{z}_{1} z_{3}\left(z_{1} \bar{\mu}-\bar{z}_{1} \mu\right)+z_{2} \bar{z}_{3}\left(z_{2} \bar{\mu}-\bar{z}_{2} \mu\right)\right) \\
& B_{2 \overline{2}}=\left|z_{3}\right|^{-2}\left(z_{2} \bar{z}_{3}-z_{3} \bar{z}_{2}\right)\left(z_{1} \bar{\mu}-\bar{z}_{1} \mu\right)
\end{aligned}
$$

and

$$
z_{3}=\sqrt{\left(\epsilon^{2}-\mu^{2}\right)-\left(z_{1}^{2}+z_{2}^{2}\right)} .
$$

The components of the Kähler two-forms $J$ are given by:

$$
\begin{aligned}
& \hat{\eta}_{5}=\eta_{5} \mid\left(z_{4}=\mu\right)=J_{1 \overline{1}}^{\prime} d z_{1} \wedge d \bar{z}_{1}+J_{1 \overline{2}}^{\prime} d z_{1} \wedge d \bar{z}_{2}+J_{2 \overline{1}}^{\prime} d z_{2} \wedge d \bar{z}_{1}+J_{2 \overline{2}}^{\prime} d z_{2} \wedge d \bar{z}_{2}, \\
& \hat{\eta}_{4}=\eta_{4}\left(z_{4}=\mu\right)=J_{1 \overline{1}}^{\prime \prime} d z_{1} \wedge d \bar{z}_{1}+J_{1 \overline{2}}^{\prime \prime} d z_{1} \wedge d \bar{z}_{2}+J_{2 \overline{1}}^{\prime \prime} d z_{2} \wedge d \bar{z}_{1}+J_{2 \overline{2}}^{\prime \prime} d z_{2} \wedge d \bar{z}_{2} .
\end{aligned}
$$

Here the various functions are given by:

$$
\begin{aligned}
& J_{1 \overline{1}}^{\prime}=1+\frac{\left|z_{1}\right|^{2}}{\left|z_{3}\right|^{2}}, \\
& J_{1 \overline{2}}^{\prime}=\frac{z_{1} \bar{z}_{2}}{\left|z_{3}\right|^{2}}, \\
& J_{1 \overline{1}}^{\prime \prime}=-\frac{\left(z_{1} \bar{z}_{3}-z_{3} \bar{z}_{1}\right)^{2}}{\left|z_{3}\right|^{2}}, \\
& J_{1 \overline{2}}^{\prime \prime}=J_{2 \overline{1}}^{\prime \prime}=-\frac{\left(z_{1} \bar{z}_{3}-z_{3} \bar{z}_{1}\right)\left(z_{2} \bar{z}_{3}-z_{3} \bar{z}_{2}\right)}{\left|z_{3}\right|^{2}} .
\end{aligned}
$$

By either tedious or computer assisted algebra, one now obtains

$$
\begin{aligned}
& \hat{\eta}_{5} \wedge \hat{\eta}_{1}=0 \\
& \hat{\eta}_{4} \wedge \hat{\eta}_{1}=0 .
\end{aligned}
$$


From equations (4.8) and (4.9), we can confirm that the SUSY condition (3.3) is satisfied by the holomorphic embedding (4.1), as noticed in [33]. Moreover as $\hat{\mathcal{F}}=\hat{B}_{2}$, the Bianchi identity (3.4) is also trivially satisfied.

\section{$4.2 w$-Embedding}

Let us now consider the holomorphic embedding given in [34]:

$$
w_{1}=\frac{z_{1}+i z_{2}}{\sqrt{2}}=\mu, \quad \mu \in \mathbb{C} .
$$

This embedding breaks the $\mathrm{SO}(4) \cong \mathrm{SU}(2) \times \mathrm{SU}(2)$ rotational symmetry of the deformed conifold to the $\mathrm{U}(1)$ subgroup which rotates $w_{3}$ and $w_{4}$ by opposite phases. By combining (4.10) with the defining equation of the deformed conifold, we can also deduce that

$$
w_{2}=\frac{z_{1}-i z_{2}}{\sqrt{2}}=\frac{\epsilon^{2}-\left(z_{3}^{2}+z_{4}^{2}\right)}{2 \mu},
$$

which leaves us only $\left\{z_{3}, z_{4}\right\}$ or $\left\{w_{3}, w_{4}\right\}$ and their complex conjugates as the independent variables parameterizing the four cycle wrapped by the D7 brane. In this case we shall first demonstrate that supersymmetry is broken and then using numerical methods construct the additional world volume flux which restores it.

To check the supersymmetry condition (3.5), the pull-back of the NS-NS two form field $\hat{B}_{2}=b(\rho) \hat{\eta}_{1}$ can be obtained by calculating

$$
\hat{\eta}_{1}=\left.\eta_{1}\right|_{\left(w_{1}=\mu\right)}=B_{3 \overline{3}} d z_{3} \wedge d \bar{z}_{3}+B_{3 \overline{4}} d z_{3} \wedge d \bar{z}_{4}+B_{4 \overline{3}} d z_{4} \wedge d \bar{z}_{3}+B_{4 \overline{4}} d z_{4} \wedge d \bar{z}_{4},
$$

where the various components are:

$$
\begin{aligned}
& B_{3 \overline{3}}= i\left\{\left(z_{4} \bar{z}_{3}-z_{3} \bar{z}_{4}\right)\left(1+\frac{\left|z_{3}\right|^{2}}{|\mu|^{2}}\right)+\frac{z_{3} z_{4}}{\sqrt{2} \mu}\left(\bar{z}_{1}+i \bar{z}_{2}\right)-\frac{\bar{z}_{3} \bar{z}_{4}}{\sqrt{2} \bar{\mu}}\left(z_{1}-i z_{2}\right)\right\}, \\
& B_{3 \overline{4}}= i\left\{\left(z_{4} \bar{z}_{3}-z_{3} \bar{z}_{4}\right)\left(\frac{z_{3} \bar{z}_{4}}{|\mu|^{2}}\right)-i\left(z_{1} \bar{z}_{2}-\bar{z}_{1} z_{2}\right)\right. \\
&\left.+\left(\left|z_{3}\right|^{2}+\left|z_{4}\right|^{2}\right)-\frac{z_{3}^{2}}{\sqrt{2} \mu}\left(\bar{z}_{1}+i \bar{z}_{2}\right)-\frac{\bar{z}_{4}^{2}}{\sqrt{2} \bar{\mu}}\left(z_{1}-i z_{2}\right)\right\}, \\
& B_{4 \overline{3}}=i\left\{\left(z_{4} \bar{z}_{3}-z_{3} \bar{z}_{4}\right)\left(\frac{z_{4} \bar{z}_{3}}{|\mu|^{2}}\right)+i\left(z_{1} \bar{z}_{2}-\bar{z}_{1} z_{2}\right)\right. \\
&\left.-\left(\left|z_{3}\right|^{2}+\left|z_{4}\right|^{2}\right)+\frac{z_{4}^{2}}{\sqrt{2} \mu}\left(\bar{z}_{1}+i \bar{z}_{2}\right)+\frac{\bar{z}_{3}^{2}}{\sqrt{2} \bar{\mu}}\left(z_{1}-i z_{2}\right)\right\}, \\
& B_{4 \overline{4}}=i\left\{\left(z_{4} \bar{z}_{3}-z_{3} \bar{z}_{4}\right)\left(1+\frac{\left|z_{4}\right|^{2}}{|\mu|^{2}}\right)-\frac{z_{3} z_{4}}{\sqrt{2} \mu}\left(\bar{z}_{1}+i \bar{z}_{2}\right)+\frac{\bar{z}_{3} \bar{z}_{4}}{\sqrt{2} \bar{\mu}}\left(z_{1}-i z_{2}\right)\right\} .
\end{aligned}
$$

Notice that $\hat{\eta}_{1}$ is now only invariant under the $\mathrm{U}(1)$ subgroup, and the invariance can be made manifest by changing into the coordinates $\left\{w_{3}, w_{4}, \bar{w}_{3}, \bar{w}_{4}\right\}$.

The components for the pull-back of the Kähler form $\hat{J}=\mathcal{K}^{\prime}(\rho) \hat{\eta}_{5}+\mathcal{K}^{\prime \prime}(\rho) \hat{\eta}_{4}$ can also be obtained by calculating:

$$
\begin{aligned}
& \hat{\eta}_{5}=\eta_{5} \mid\left(w_{1}=\mu\right)=J_{3 \overline{3}}^{\prime} d z_{3} \wedge d \bar{z}_{3}+J_{3 \overline{4}}^{\prime} d z_{3} \wedge d \bar{z}_{4}+J_{4 \overline{3}}^{\prime} d z_{4} \wedge d \bar{z}_{3}+J_{4 \overline{4}}^{\prime} d z_{4} \wedge d \bar{z}_{4} \\
& \hat{\eta}_{4}=\left.\eta_{4}\right|_{\left(w_{1}=\mu\right)}=J_{3 \overline{3}}^{\prime \prime} d z_{3} \wedge d \bar{z}_{3}+J_{3 \overline{4}}^{\prime \prime} d z_{3} \wedge d \bar{z}_{4}+J_{4 \overline{3}}^{\prime \prime} d z_{4} \wedge d \bar{z}_{3}+J_{4 \overline{4}}^{\prime \prime} d z_{4} \wedge d \bar{z}_{4}
\end{aligned}
$$


Here the various functions are given by:

$$
\begin{aligned}
& J_{3 \overline{3}}^{\prime}=1+\frac{\left|z_{3}\right|^{2}}{|\mu|^{2}}, \quad J_{4 \overline{4}}^{\prime}=1+\frac{\left|z_{4}\right|^{2}}{|\mu|^{2}}, \quad J_{3 \overline{4}}^{\prime}=\frac{z_{3} \bar{z}_{4}}{|\mu|^{2}}, \quad J_{4 \overline{3}}^{\prime}=\frac{z_{4} \bar{z}_{3}}{|\mu|^{2}} . \\
& J_{3 \overline{3}}^{\prime \prime}=S_{3} \bar{S}_{3}, \quad J_{3 \overline{4}}^{\prime \prime}=S_{3} \bar{S}_{4}, \quad J_{4 \overline{3}}^{\prime \prime}=S_{4} \bar{S}_{3}, \quad J_{4 \overline{4}}^{\prime \prime}=S_{4} \bar{S}_{4}, \\
& S_{3}=\bar{z}_{3}-\frac{\left(\bar{\epsilon}^{2}-\left(\bar{z}_{3}^{2}+\bar{z}_{4}^{2}\right)\right) z_{3}}{2|\mu|^{2}}, \quad S_{4}=\bar{z}_{4}-\frac{\left(\bar{\epsilon}^{2}-\left(\bar{z}_{3}^{2}+\bar{z}_{4}^{2}\right)\right) z_{4}}{2|\mu|^{2}}
\end{aligned}
$$

Similarly, $\hat{\eta}_{4}$ and $\hat{\eta}_{5}$ are now only invariant under the $\mathrm{U}(1)$ isometry rotating the phase of $w_{3} / w_{4}$. Given the expressions for $\hat{B}_{2}$, and the pull-back of the Kähler form $J$ for the embedding (4.10), we can now calculate the wedge products explicitly and check if the supersymmetric condition (3.3) is satisfied. Let us calculate them in turn:

$$
\begin{aligned}
\hat{\eta}_{5} \wedge \hat{\eta}_{1} & =i \frac{\left(z_{3} \bar{z}_{4}-z_{4} \bar{z}_{3}\right)}{|\mu|^{2}}\left(\left|z_{1}\right|^{2}+\left|z_{2}\right|^{2}+\left|z_{3}\right|^{2}+\left|z_{4}\right|^{2}\right) d \Omega \\
& =-\frac{|\epsilon|^{4} \sinh \rho \cosh \rho \sin \left(\frac{\theta_{1}+\theta_{2}}{2}\right) \sin \left(\frac{\theta_{1}-\theta_{2}}{2}\right)}{|\mu|^{2}} d \Omega
\end{aligned}
$$

with $d \Omega=d z_{3} \wedge d z_{4} \wedge d \bar{z}_{3} \wedge d \bar{z}_{4}$, and we have used the explicit coordinates on the deformed conifold given in appendix A. Notice that the overall expression in (4.22) is in fact real as $i\left(z_{3} \bar{z}_{4}-z_{4} \bar{z}_{3}\right)=2 \operatorname{Im}\left(z_{4} \bar{z}_{3}\right)$. Similarly

$$
\begin{aligned}
\hat{\eta}_{4} \wedge \hat{\eta}_{1} & =-\frac{i\left(z_{3} \bar{z}_{4}-z_{4} \bar{z}_{3}\right)}{|\mu|^{2}} \sum_{i \neq j=1}^{4}\left(z_{i} \bar{z}_{j}-z_{j} \bar{z}_{i}\right)^{2} d \Omega \\
& =-\frac{|\epsilon|^{6} \sinh ^{3} \rho \sin \left(\frac{\theta_{1}+\theta_{2}}{2}\right) \sin \left(\frac{\theta_{1}-\theta_{2}}{2}\right)}{|\mu|^{2}} d \Omega .
\end{aligned}
$$

Here we notice that the summation in (4.23) is in fact positive definite, since each term in the summation (including the negative sign) is nothing but $\left(\operatorname{Im}\left(z_{i} \bar{z}_{j}\right)\right)^{2}$. Combining (4.22) and (4.23), we can finally write down the expression for $\hat{J} \wedge \hat{B}_{2}$ (including the scalar functions):

$$
\hat{J} \wedge \hat{B}_{2}=-\mathcal{K}^{\prime}(\rho) b(\rho) \frac{|\epsilon|^{4} \sin \left(\frac{\theta_{1}+\theta_{2}}{2}\right) \sin \left(\frac{\theta_{1}-\theta_{2}}{2}\right)}{3|\mu|^{2}} \sinh ^{2} \rho\left(\frac{5+\cosh (2 \rho)}{\sinh (2 \rho)-2 \rho}\right) d \Omega,
$$

where the scalar functions $\mathcal{K}^{\prime}(\rho)$ and $b(\rho)$ are as given in (2.32) and (2.31). The expression (4.24) is manifestly non-vanishing and we conclude that in the absence of additional worldvolume flux $F_{2}$, supersymmetry is broken.

\subsection{Restoring supersymmetry with worldvolume flux}

Because $\hat{B}_{2}$ is not primitive, it is clear that D7-branes in the $w$-embedding are not supersymmetric without the addition of worldvolume flux. In this section we will attempt to construct the necessary flux. After setting up the problem, we explore the asymptotic 
behavior of the flux in sections 4.3.1-4.3.4 before turning to a numerical solution in section 4.3.5. The results are summarized in section 4.3.6. Part of our solution agrees in appropriate asymptotic regimes with a previous proposal in [41], with some corrections which we describe in detail.

Our strategy is to first find a basis of 2-forms on the D7 worldvolume which are $(1,1)$ and primitive. Then we take a linear combination of these forms and impose the Bianchi identity, which gives a system of partial differential equations for the coefficients of the basis forms, and we proceed to solve this system.

To make the calculations more tractable we will suppose the parameter $\mu$ is much greater than the deformation parameter $\epsilon$ so that we may work in the KT region of the deformed conifold. In this region, the background geometry is simply that of the warped conifold with no deformation but with nontrivial background fluxes. The advantage of taking this limit lies in the symmetries of the background. In taking $w_{1}=\mu$ on the deformed conifold the isometries of $T^{1,1}$ are broken down to a single $\mathrm{U}(1)$, but in the KT limit the isometry is $\mathrm{U}(1) \times \mathrm{U}(1)$. In addition to the $\mathrm{U}(1)$ on the deformed conifold which rotates $w_{3}$ and $w_{4}$ by opposite phases, there is an extra $\mathrm{U}(1)$ in the KT region which acts as $w_{2} \rightarrow e^{2 i \alpha} w_{2}, w_{3} \rightarrow e^{i \alpha} w_{3}, w_{4} \rightarrow e^{i \alpha} w_{4}$. The $\mathrm{U}(1)^{2}$ isometry implies that instead of having a four-variable problem, we can use the isometry to eliminate the azimuthal angles, reducing our problem to a problem of two spatial variables.

It is useful to express our embedding in terms of explicit coordinates on the conifold. Upon restricting to $w_{1}=r^{3 / 2} e^{i / 2\left(\psi-\phi_{1}-\phi_{2}\right)} \sin \frac{\theta_{1}}{2} \sin \frac{\theta_{2}}{2}=\mu$, we see that we may eliminate

$$
r=\left(\frac{\mu}{\sin \frac{\theta_{1}}{2} \sin \frac{\theta_{2}}{2}}\right)^{2 / 3}, \quad \psi=\phi_{1}+\phi_{2},
$$

and take $\mu$ to be real. In terms of these angular coordinates, large $r$ corresponds to $\theta_{i} \rightarrow 0$ and when $\theta_{1}=\theta_{2}=\pi$ we are at the minimal radius to which the D7-brane extends.

When pulled back to the locus $w_{1}=\mu$, the $\Omega_{i j}$ in (2.6) may be written as

$$
\begin{aligned}
\Omega_{11} & =-2 i \mu^{2} \frac{d w_{4} \wedge d \bar{w}_{4}}{\left(\left|w_{4}\right|^{2}+\mu^{2}\right)^{2}} \\
\Omega_{22} & =-2 i \mu^{2} \frac{d w_{3} \wedge d \bar{w}_{3}}{\left(\left|w_{3}\right|^{2}+\mu^{2}\right)^{2}} \\
\Omega_{12}+\Omega_{21} & =-2 i \mu^{2} \frac{w_{3} \bar{w}_{4} d w_{4} \wedge d \bar{w}_{3}+w_{4} \bar{w}_{3} d w_{3} \wedge d \bar{w}_{4}}{\left|w_{3}\right|\left|w_{4}\right|\left(\left|w_{3}\right|^{2}+\mu^{2}\right)\left(\left|w_{4}\right|^{2}+\mu^{2}\right)}
\end{aligned}
$$

To check primitivity, we need the Kahler form (up to a factor) pulled back to the D7 brane worldvolume:

$$
\hat{J} \propto Q_{1} \Omega_{11}+Q_{2} \Omega_{22}+\cot \frac{\theta_{1}}{2} \cot \frac{\theta_{2}}{2}\left(\Omega_{12}+\Omega_{21}\right)
$$

where

$$
Q_{i}=\frac{3}{2}+\cot ^{2} \frac{\theta_{i}}{2}
$$


There are two simple anti-self-dual $(1,1)$ forms which are relevant to us:

$$
\begin{aligned}
& X_{1}=\Omega_{11}+\frac{1}{2} \tan \frac{\theta_{1}}{2} \tan \frac{\theta_{2}}{2} Q_{2}\left(\Omega_{21}+\Omega_{12}\right) \\
& X_{2}=\Omega_{22}+\frac{1}{2} \tan \frac{\theta_{1}}{2} \tan \frac{\theta_{2}}{2} Q_{1}\left(\Omega_{21}+\Omega_{12}\right)
\end{aligned}
$$

There is also a third anti-self-dual $(1,1)$ form, but it plays no role in the later calculations:

$$
X_{3}=d \theta_{1} \wedge d \theta_{2}-\sin \theta_{1} \sin \theta_{2} d \phi_{1} \wedge d \phi_{2} .
$$

It is not hard to see that any component of $X_{3}$ in $\hat{\mathcal{F}}$ will either induce a violation of the Bianchi identity or will be singular.

We can take the following linear combinations of $X_{1}$ and $X_{2}$ which have nice properties:

$$
\begin{aligned}
& P=Q_{1} X_{1}-Q_{2} X_{2}=Q_{1} \Omega_{11}-Q_{2} \Omega_{22}, \\
& Q=X_{1}-X_{2} .
\end{aligned}
$$

The form $P$ is closed, $d P=0$, while $Q$ has a particularly simple exterior derivative:

$$
d Q=\frac{d \theta_{1}}{\sin \theta_{1}} \wedge \Omega_{22}-\frac{d \theta_{2}}{\sin \theta_{2}} \wedge \Omega_{11} .
$$

We will use $P$ and $Q$ as basis two-forms for the worldvolume flux, and search for an anti-self-dual form $\hat{\mathcal{F}}=\alpha\left(\theta_{1}, \theta_{2}\right) P+\beta\left(\theta_{1}, \theta_{2}\right) Q$ which satisfies the Bianchi identity. It is enlightening to write the forms $P, Q$ explicitly in complex coordinates:

$$
\begin{aligned}
\frac{P}{2 i \mu^{2}}= & \left(\frac{3}{2}+\frac{\left|w_{3}\right|^{2}}{\mu^{2}}\right) \frac{d w_{3} \wedge d \bar{w}_{3}}{\left(\left|w_{3}\right|^{2}+\mu^{2}\right)^{2}}-\left(\frac{3}{2}+\frac{\left|w_{4}\right|^{2}}{\mu^{2}}\right) \frac{d w_{4} \wedge d \bar{w}_{4}}{\left(\left|w_{4}\right|^{2}+\mu^{2}\right)^{2}} \\
\frac{Q}{2 i \mu^{2}}= & \frac{d w_{3} \wedge d \bar{w}_{3}}{\left(\left|w_{3}\right|^{2}+\mu^{2}\right)^{2}}-\frac{d w_{4} \wedge d \bar{w}_{4}}{\left(\left|w_{4}\right|^{2}+\mu^{2}\right)^{2}} \\
& \quad+\left(\frac{\mu^{2}}{\left|w_{3}\right|^{2}}-\frac{\mu^{2}}{\left|w_{4}\right|^{2}}\right) \frac{w_{3} \bar{w}_{4} d w_{4} \wedge d \bar{w}_{3}+w_{4} \bar{w}_{3} d w_{3} \wedge d \bar{w}_{4}}{\left(\left|w_{3}\right|^{2}+\mu^{2}\right)\left(\left|w_{4}\right|^{2}+\mu^{2}\right)}
\end{aligned}
$$

By counting powers of $w_{3,4}$, we see that in the limit $w_{3}, w_{4} \rightarrow \infty, Q$ is non-singular while $P$ is potentially log-singular. On the other hand, when $w_{3}$ or $w_{4}$ vanishes, $P$ is non-singular while $Q$ can be log-singular.

If one prefers to parameterize the D7-brane worldvolume by the angular coordinates $\left(\theta_{1}, \phi_{1}, \theta_{2}, \phi_{2}\right)$, the basis forms may be written as

$$
\begin{gathered}
P=\left(\frac{3}{2}+\cot ^{2} \frac{\theta_{1}}{2}\right) d \theta_{1} \wedge \sin \theta_{1} d \phi_{1}-\left(\frac{3}{2}+\cot ^{2} \frac{\theta_{2}}{2}\right) d \theta_{2} \wedge \sin \theta_{2} d \phi_{2} \\
Q=\frac{1}{2}\left(\tan \frac{\theta_{1}}{2} \cot \frac{\theta_{2}}{2}-\cot \frac{\theta_{1}}{2} \tan \frac{\theta_{2}}{2}\right)\left(d \theta_{2} \wedge \sin \theta_{1} d \phi_{1}+d \theta_{1} \wedge \sin \theta_{2} d \phi_{2}\right) \\
+d \theta_{1} \wedge \sin \theta_{1} d \phi_{1}-d \theta_{2} \wedge \sin \theta_{2} d \phi_{2} .
\end{gathered}
$$

Let us now turn to the Bianchi identity which $\hat{\mathcal{F}}$ must satisfy. The B-field in the Klebanov-Tseytlin geometry is $B_{2}=\frac{3 g_{s} M \alpha^{\prime}}{2} \log \frac{r}{r_{0}} \omega_{2}$, so that pulling back $H_{3}=d B_{2}$ to the D7-brane worldvolume we have

$$
d \hat{\mathcal{F}}=\hat{H}_{3}=-\frac{g_{s} M \alpha^{\prime}}{2}\left(\cot \frac{\theta_{1}}{2} d \theta_{1}+\cot \frac{\theta_{2}}{2} d \theta_{2}\right) \wedge \omega_{2}
$$


Plugging in the ansatz $\hat{\mathcal{F}}=\alpha\left(\theta_{1}, \theta_{2}\right) P+\beta\left(\theta_{1}, \theta_{2}\right) Q$, we obtain a first-order system of partial differential equations for $\alpha\left(\theta_{1}, \theta_{2}\right)$ and $\beta\left(\theta_{1}, \theta_{2}\right)$ :

$$
\begin{aligned}
& \partial_{\theta_{1}} \alpha=-\frac{S}{Q_{2}} \partial_{\theta_{2}} \beta-\frac{1}{Q_{2}} \partial_{\theta_{1}} \beta+\frac{1}{Q_{2} \sin \theta_{1}} \beta+\frac{k}{Q_{2}} \cot \frac{\theta_{1}}{2}, \\
& \partial_{\theta_{2}} \alpha=\frac{S}{Q_{1}} \partial_{\theta_{1}} \beta-\frac{1}{Q_{1}} \partial_{\theta_{2}} \beta+\frac{1}{Q_{1} \sin \theta_{2}} \beta+\frac{k}{Q_{1}} \cot \frac{\theta_{2}}{2} .
\end{aligned}
$$

where we have

$$
S=\frac{\cos \theta_{2}-\cos \theta_{1}}{\sin \theta_{1} \sin \theta_{2}}, \quad k=-\frac{g_{s} M \alpha^{\prime}}{4}
$$

This system of differential equations is challenging to solve but we will show that numerical methods combined with some analytic tricks will allow us to find a solution. The system with two functions can be converted to a second order partial differential equation for the function $\beta\left(\theta_{1}, \theta_{2}\right)$ by differentiating and eliminating $\partial_{\theta_{1}} \partial_{\theta_{2}} \alpha$. Explicitly, the second order equation of interest takes the form

$$
\begin{gathered}
{\left[\partial_{\theta_{1}} \frac{S}{Q_{1}} \partial_{\theta_{1}}+\partial_{\theta_{2}} \frac{S}{Q_{2}} \partial_{\theta_{2}}-\partial_{\theta_{1}} \frac{1}{Q_{1}} \partial_{\theta_{2}}+\partial_{\theta_{2}} \frac{1}{Q_{2}} \partial_{\theta_{1}}+\frac{1}{Q_{1} \sin \theta_{2}} \partial_{\theta_{1}}-\frac{1}{Q_{2} \sin \theta_{1}} \partial_{\theta_{2}}\right.} \\
\left.+\frac{1}{\sin \theta_{2}} \partial_{\theta_{1}}\left(\frac{1}{Q_{1}}\right)-\frac{1}{\sin \theta_{1}} \partial_{\theta_{2}}\left(\frac{1}{Q_{2}}\right)\right] \beta\left(\theta_{1}, \theta_{2}\right) \\
=-k\left(\cot \frac{\theta_{2}}{2} \partial_{\theta_{1}}\left(\frac{1}{Q_{1}}\right)-\cot \frac{\theta_{1}}{2} \partial_{\theta_{2}}\left(\frac{1}{Q_{2}}\right)\right)
\end{gathered}
$$

Our main task in the following subsections will be to solve this complicated equation.

Once we have solved for $\beta\left(\theta_{1}, \theta_{2}\right)$, we can then find the second function $\alpha\left(\theta_{1}, \theta_{2}\right)$ by the following procedure. From the system (4.42), we construct the quantity $\left(\partial_{\theta_{1}}^{2}+\partial_{\theta_{2}}^{2}\right) \alpha$. This procedure results in a standard Poisson-type equation for $\alpha\left(\theta_{1}, \theta_{2}\right)$ with a complicated source term which depends on the function $\beta\left(\theta_{1}, \theta_{2}\right)$.

\subsubsection{Boundary conditions and finiteness}

Before turning to the numerical analysis of the flux equations, we need to identify the boundary conditions which we expect the function $\beta\left(\theta_{1}, \theta_{2}\right)$ to satisfy (Recall that the angular coordinates are defined over the range $(0, \pi)$.) We claim that there is a unique set of physical boundary conditions on the supersymmetry-restoring flux. For the function $\beta$, the boundary conditions turn out to be compatible in a certain large-radius limit with the proposal of Benini [41].

The primary physical constraint on $\beta$ is that we expect its contribution to the flux, $\beta Q$, to be finite. One then finds that the allowed boundary behavior of $\beta$ is tied to the asymptotic properties of the two-form $Q$. There are three important regimes to consider:

- $\theta_{1}=\pi$ or $\theta_{2}=\pi$ : When one or the other of the angles $\theta_{i}=\pi$, the only physical choice of boundary conditions is Dirichlet:

$$
\beta\left(\pi, \theta_{2}\right)=\beta\left(\theta_{1}, \pi\right)=0 .
$$


The simplest argument for this comes from examining the basis two-form $Q$ in (4.38). For example, in the limit $\theta_{1} \rightarrow \pi$, we have $\left|w_{4}\right| \rightarrow 0$ so the basis form $Q$ exhibits a logarithmic divergence. To exclude this singular flux, we must set $\beta=0$.

- $\theta_{2} \rightarrow 0$ with $\theta_{1}>0$, or vice versa: In this limit, $\left|w_{4}\right| \rightarrow \infty$ with $\left|w_{3}\right|$ finite, and one sees from (4.38) that $Q$ is $O(1)$. Thus the only constraint in this limit is that $\beta$ is finite.

- $\theta_{1}, \theta_{2} \rightarrow 0$ simultaneously: In this limit, $Q$ actually vanishes as $1 /|w|^{2}$, with $\left|w_{3,4}\right| \rightarrow$ $\infty$. Then finiteness of the flux only requires that $\beta$ grows no faster than $\frac{1}{\sin ^{4} \theta_{i}}$ as $\theta_{i} \rightarrow 0$.

To specify the large radius (small $\theta$ ) boundary conditions precisely, we will now study the second and third limiting cases in detail. The second case may be usefully regarded as a "factorization" limit in which the D7-brane worldvolume splits into two branches. Along one branch we have $\theta_{1} \rightarrow 0$ and along the other, we have $\theta_{2} \rightarrow 0$. The two branches connect when both $\theta_{1}, \theta_{2} \rightarrow 0$, corresponding to the third case, which represents an "interpolating" limit between the two branches.

For the second function $\alpha$, the associated asymptotics may be studied similarly. The basis form $P$ is regular in the interior of the D7-brane worldvolume, but has logarithmic singularities when $\theta_{1}$ or $\theta_{2}$ vanishes. This logarithm is physically acceptable, as it is compatible with the log growth of the fluxes in the KT solution. Therefore when either angle vanishes, we require that $\alpha$ grows no faster than $\log r$. On the other hand, in the interior of the brane, we expect $\alpha$ to be finite.

\subsubsection{Large radius "factorization" limit}

Let us take the limit $\theta_{2} \rightarrow 0$, with $\theta_{1}$ held fixed. The various terms in (4.44) have the behavior

$$
\begin{aligned}
S & \rightarrow \tan \frac{\theta_{1}}{2} \frac{1}{\theta_{2}} \\
Q_{2} & \rightarrow \frac{4}{\theta_{2}^{2}}
\end{aligned}
$$

Multiplying the equation for $\beta$ by $\theta_{2}$ and taking the limit as $\theta_{2} \rightarrow 0$, we see that (4.44) reduces to the following, discarding terms of order $\theta_{2}^{2}$ :

$$
\begin{gathered}
{\left[\partial_{\theta_{1}} \frac{1}{Q_{1}} \tan \frac{\theta_{1}}{2} \partial_{\theta_{1}}+\frac{1}{4} \tan \frac{\theta_{1}}{2} \theta_{2} \partial_{\theta_{2}} \theta_{2} \partial_{\theta_{2}}-\partial_{\theta_{1}} \frac{1}{Q_{1}} \theta_{2} \partial_{\theta_{2}}+\frac{1}{Q_{1}} \partial_{\theta_{1}}+\partial_{\theta_{1}}\left(\frac{1}{Q_{1}}\right)\right] \beta} \\
=-2 k \partial_{\theta_{1}}\left(\frac{1}{Q_{1}}\right)
\end{gathered}
$$

Unfortunately this differential equation is not separable, but it does have homogeneous scaling with $\theta_{2}$ - all terms are of zeroth order.

In the previous subsection we argued that in this limit $\beta$ should be finite. Thus the leading small $\theta_{2}$ behavior should be for $\beta$ to be constant as a function of $\theta_{2}$. The 
equation (4.48) simplifies to

$$
\partial_{\theta_{1}} \frac{1}{Q_{1}} \tan \frac{\theta_{1}}{2} \partial_{\theta_{1}} \beta+\partial_{\theta_{1}}\left(\frac{\beta}{Q_{1}}\right)=-2 k
$$

and the solution is

$$
\beta=-2 k+\frac{c_{1}}{2 \sin ^{2} \frac{\theta_{1}}{2}}\left(\cos \theta_{1}-8 \log \sin \frac{\theta_{1}}{2}\right)+\frac{c_{2}}{2 \sin ^{2} \frac{\theta_{1}}{2}}
$$

with two integration constants which we need to determine. We may fix one constant by requiring consistency with the Dirichlet boundary condition $\beta\left(\theta_{1}=\pi\right)=0$, which is satisfied if

$$
-2 k-\frac{c_{1}}{2}+\frac{c_{2}}{2}=0 .
$$

We will be able to obtain a second constraint after we have performed the analysis in the next subsection.

Now we can substitute into the first order equations to solve for $\alpha$. In the small $\theta_{2}$ limit these reduce to

$$
\begin{aligned}
\partial_{\theta_{1}} \alpha & =-\frac{1}{4} \tan \frac{\theta_{1}}{2} \theta_{2} \partial_{\theta_{2}} \beta \\
\theta_{2} \partial_{\theta_{2}} \alpha & =\frac{\tan \frac{\theta_{1}}{2}}{Q_{1}} \partial_{\theta_{1}} \beta-\frac{1}{Q_{1}} \theta_{2} \partial_{\theta_{2}} \beta+\frac{1}{Q_{1}} \beta+\frac{2 k}{Q_{1}} .
\end{aligned}
$$

The first equation reduces in our limit to $\partial_{\theta_{1}} \alpha=0$ because $\beta$ is independent of $\theta_{2}$. The second equation with $\beta$ plugged in gives $\alpha \sim-2 c_{1} \log \theta_{2}$.

\subsubsection{Large radius "interpolating" limit}

When $\theta_{1}$ and $\theta_{2}$ are simultaneously small, the asymptotic analysis of section 4.3 .2 breaks down. For example, there can be terms of the form $\frac{\theta_{2}}{\theta_{1}}$ which we discarded in the strict $\theta_{2} \rightarrow$ 0 limit but are order one when the two angles are small simultaneously. This immediately raises the question of whether it is possible for the solutions along the two branches $\theta_{1}=0$ and $\theta_{2}=0$ to be mutually consistent when both angles are small, so that the flux smoothly interpolates between the two branches. In the following analysis we answer this question in the affirmative.

To proceed, we have found the following change of variables to be useful:

$$
\begin{aligned}
& u=-\log \left(\sin \frac{\theta_{1}}{2} \sin \frac{\theta_{2}}{2}\right) \\
& v=\log \left(\frac{\sin \frac{\theta_{1}}{2}}{\sin \frac{\theta_{2}}{2}}\right)
\end{aligned}
$$

where $u$ is clearly related to the original radial coordinate $r$ as in (4.25) The natural limit to take with both of the $\theta_{i}$ small is simply

$$
u \rightarrow \infty, \quad v \text { held fixed. }
$$


Now we turn to the decoupled second order equation (4.44) for $\beta$, in the $u$ and $v$ coordinates. We may expand the resulting equation in powers of $e^{-u}$ for large $u$, in the form

$$
\left(\mathcal{O}_{0}+e^{-u} \mathcal{O}_{1}+e^{-2 u} \mathcal{O}_{2}+\ldots\right) \beta=g_{0}+e^{-u} g_{1}+\ldots
$$

with homogeneous and inhomogeneous parts separated. It is tedious but straightforward to find (multiplying the equation obtained from (4.44) by an overall factor to reduce clutter)

$$
\begin{aligned}
\mathcal{O}_{0} & =\frac{\partial^{2}}{\partial v^{2}}+2 \operatorname{coth} v \frac{\partial}{\partial v}+1 \\
\mathcal{O}_{1} & =\frac{\cosh v}{4}\left(3 \frac{\partial^{2}}{\partial u^{2}}-2 \frac{\partial}{\partial u}-\frac{\partial^{2}}{\partial v^{2}}\right)+\frac{3}{2} \sinh v \frac{\partial^{2}}{\partial u \partial v} \\
& \quad-\frac{1}{2}\left(7 \sinh v+\frac{1}{\sinh v}\right) \frac{\partial}{\partial v}-2 \cosh v \\
g_{0} & =-2 k \\
g_{1} & =4 k \cosh v
\end{aligned}
$$

In principle one can now expand $\beta$ in powers of $e^{-u}$ and construct a solution iteratively, order by order in $e^{-u}$.

At the zeroth order in the expansion, we encounter a small surprise: in the large $u$ limit, the leading homogeneous part of the equation for $\beta$ reduces to an ordinary differential equation,

$$
\mathcal{O}_{0} \beta_{h}=0
$$

This equation is easily solved:

$$
\beta_{h}=f_{1}(u) \frac{v}{\sinh v}+f_{2}(u) \frac{1}{\sinh v}
$$

Demanding that $\beta$ is non-singular at $v=0$ sets $f_{2}=0$.

At leading order, $f_{1}(u)$ can be any function of $u$, but in the full solution its form must be compatible with all the other asymptotics we have identified. This eliminates most possibilities for $f_{1}$. For example, a natural ansatz one might try is to set $\beta=-2 k+f_{1}(u) \frac{v}{\sinh v}+O\left(e^{-u}\right)$ with $f_{1}(u)$ of zeroth order in $e^{-u}$, so that the leading homogeneous solution and the leading particular solution are of the same order. However, a little manipulation shows that this ansatz must be wrong, because the homogeneous part vanishes when $v \rightarrow \pm \infty$. The resulting function cannot possibly satisfy the Dirichlet boundary condition (4.45).

What we need is a form for the function $f_{1}(u)$ so that the homogeneous piece $\beta_{h}$ approaches a constant in the limit $u \rightarrow \infty, v \rightarrow \pm \infty$ (which corresponds to $\left(\theta_{1}, \theta_{2}\right) \rightarrow(0, \pi)$ or $(\pi, 0)$. There is only one such function which suffices, up to subleading terms:

$$
\beta_{h}=b e^{u}\left(\frac{1}{u} \frac{v}{\sinh v}\right)+\ldots
$$

Note that the growth of this $\beta_{h}$ for $u \rightarrow \infty$ is sufficiently slow to be physically acceptable, as determined in section 4.3.1. At the next order in $e^{-u}$, we find that the system of equations 
is solved by

$$
\beta=\beta_{h}-2 k+O(1 / u)
$$

We will fix the coefficient $b$ in the next subsection.

Let us now turn our attention to the second function $\alpha$. The coupled first order equations for $\alpha$ and $\beta$ in the $u$ and $v$ variables, expanded to leading order in $e^{-u}$, are:

$$
\begin{aligned}
& \partial_{u} \alpha=-e^{-u}\left(\cosh v\left(\partial_{u} \beta+\beta+2 k\right)+2 \sinh v \partial_{v} \beta\right) \\
& \partial_{v} \alpha=-e^{-u}\left(\cosh v \partial_{v} \beta+\sinh v(\beta+2 k)\right)
\end{aligned}
$$

To leading order at large $u$ (and including the first subleading term in $1 / u$ ), this is solved by

$$
\alpha=\alpha_{0}-2 b \log u-b \frac{v \operatorname{coth} v}{u}+O\left(1 / u^{2}\right)
$$

Because the basis form $P$ was closed by itself, $\alpha$ can always be shifted by a constant $\alpha_{0}$.

One curious feature of the function $\beta$ which is clear from the large- $u$ expansion is that the first subleading term for small $\theta_{2}$ depends as $1 / \log \left(\sin \frac{\theta_{2}}{2}\right)$; in radial coordinates this appears as $1 / \log (r)$ rather than as a power of $r$. The function $1 / \log \left(\sin \frac{\theta_{2}}{2}\right)$ has the amusing property that although it vanishes when $\theta_{2} \rightarrow 0$, its first derivative with respect to $\theta_{2}$ diverges. We will make use of this subleading behavior in the next section.

\subsubsection{Consistent boundary conditions and subleading asymptotics}

Having found asymptotic solutions in two different large radius regimes, we now must check that these solutions are mutually consistent. Along the way, we will need to compute the next-to-leading asymptotic behavior of the solutions to our PDEs.

The first additional consistency requirement will fix the ambiguity we left in (4.50). There we had two integration constants, $c_{1}$ and $c_{2}$, with one relation given by demanding consistency with the "small radius" Dirichlet boundary condition. To fix the second integration constant, we can take the simultaneous limit $\theta_{1} \rightarrow 0$ and $\theta_{2} \rightarrow 0$ (recall that we have solved for the exact $\theta_{1}$ dependence in the small $\theta_{2}$ limit) and then compare with the results of the "interpolating" asymptotic; the results should agree. For the limit of small $\theta_{2}$, we can take (4.50) with (4.51) imposed to find

$$
\beta \sim-\left(2 k+c_{1}\right)\left(1-\frac{1}{\sin ^{2} \frac{\theta_{1}}{2}}\right)-\frac{4 c_{1}}{\sin ^{2} \frac{\theta_{1}}{2}} \log \sin \frac{\theta_{1}}{2}
$$

whereas for (4.65) written in the angular coordinates, we find

$$
\beta \sim \frac{2 b}{\sin ^{2} \frac{\theta_{1}}{2}}\left(1-2 \frac{\log \sin \frac{\theta_{1}}{2}}{\log \sin \frac{\theta_{2}}{2}}\right)-2 k
$$

(In (4.69) and (4.70) the $\sim$ is meant to imply that subleading terms of order $\left(\log \sin \frac{\theta_{2}}{2}\right)^{-1}$ have been dropped, although we have retained a term proportional to 
$\frac{\log \sin \frac{\theta_{1}}{2}}{\log \sin \frac{\theta_{2}}{2}}$.) Demanding that the terms proportional to a constant times $\frac{1}{\sin ^{2} \frac{\theta_{1}}{2}}$ and $\frac{\log \sin \frac{\theta_{1}}{2}}{\sin ^{2} \frac{\theta_{1}}{2}}$ are in agreement sets ${ }^{1}$

$$
\begin{aligned}
c_{1} & =0 \\
b & =k .
\end{aligned}
$$

Note that with $c_{1}=0$, we have

$$
\beta=2 k \cot ^{2} \frac{\theta_{1}}{2}
$$

in the leading small $\theta_{2}$ limit. This asymptotic for $\beta$ are identical to the proposal of [41]. The interested reader will find a comparison of our notations in appendix D.

To obtain a more detailed matching, and to see the leading nontrivial behavior of $\alpha$, we must go on to the first subleading order. Expanding in the natural expansion parameter, $1 / \log \theta_{2}$, we write

$$
\beta=-2 k+\beta_{0}+\frac{\beta_{1}}{\log \theta_{2}}+\frac{\beta_{2}}{\log ^{2} \theta_{2}}+\ldots
$$

The function $\beta_{0}$ was given in (4.73) and the solution for $\beta_{1}$ has the same form:

$$
\beta_{1}=\frac{c_{3}}{2 \sin ^{2} \frac{\theta_{1}}{2}}\left(\cos \theta_{1}-8 \log \sin \frac{\theta_{1}}{2}\right)+\frac{c_{4}}{2 \sin ^{2} \frac{\theta_{1}}{2}} .
$$

As with the leading order, we set the two integration constants as follows. One constant is set by demanding consistency with the small radius Dirichlet condition and the second is set by comparing with the term proportional to $\frac{\log \sin \frac{\theta_{1}}{2}}{\log \sin \frac{\theta_{2}}{2} \sin ^{2} \frac{\theta_{1}}{2}}$ in (4.70) (the other terms cannot be matched directly without working at higher order in the large- $u$ expansion, because we have discarded $1 / u$ corrections in $\beta_{h}$ which can shift these terms.) These two constraints set

$$
c_{3}=c_{4}=k
$$

so

$$
\beta_{1}=\frac{k}{\sin ^{2} \frac{\theta_{1}}{2}}\left(\cos ^{2} \frac{\theta_{1}}{2}-4 \log \sin \frac{\theta_{1}}{2}\right)
$$

Now, to find the leading behavior of $\alpha$, we plug into the the equations (4.52), (4.53) and solve. The answer is analytic but somewhat complicated:

$$
\begin{aligned}
\alpha=\alpha_{0}-2 k \log \left|\log \frac{\theta_{2}}{2}\right|+\frac{k}{\log ^{2} \theta_{2}}\left(-\log ^{2} \sin \frac{\theta_{1}}{2}+2 \log \sin \frac{\theta_{1}}{2} \log \cos \frac{\theta_{1}}{2}\right. \\
\left.+\frac{1}{2} \log \sin \frac{\theta_{1}}{2}+\frac{1}{2} \operatorname{Li}_{2}\left(\sin ^{2} \frac{\theta_{1}}{2}\right)\right)
\end{aligned}
$$

\footnotetext{
${ }^{1}$ One slightly subtle point is that in the factorization limit $\theta_{2} \rightarrow 0$, in principle we should also collect contributions at all orders of $e^{n(v-u)}$, from the point of view of the interpolating asymptotic. However, the additional contributions which we drop are not proportional to $\frac{1}{\sin ^{2} \frac{\theta_{1}}{2}}$ so they do not affect the matching of these particular terms.
} 
The leading $\log \log$ is consistent with the behavior in the large- $u$ limit of (4.68), but its appearance is perhaps a bit surprising from the perspective of the "factorization" limit in section 4.3.2. In the factorization limit, at leading order the $\log \log$ is not necessary to solve the field equations (although it could have been included), but it is actually necessary to include it to solve consistently at first subleading order.

\subsubsection{Numerical solution}

In this subsection we construct a numerical solution for the functions $\alpha$ and $\beta$ characterizing the D7-brane worldvolume flux; the numerical computation gives strong evidence that the D7-branes in the $w$-embedding are supersymmetric with the addition of this flux.

In the preceding analysis, we identified a self-consistent set of asymptotic solutions for the differential equation (4.44). The task that remains is for us to show that these asymptotics correspond to a smooth solution over the entire worldvolume of the D7-brane.

Under more fortunate circumstances, we would be able to invoke existence and uniqueness theorems for solutions of this PDE, and the task of showing that the D7-brane is supersymmetric would be complete. As it happens, the mathematical problem we must solve is not of a standard type. The second-order PDE for $\beta$ is elliptic through most of its domain, but it fails to be elliptic on the line $\theta_{1}=\theta_{2}$, where the equation vanishes identically (recall that the second order equation was obtained by differentiating and subtracting the first order equations.) We are not aware of general theorems for this type of equation. ${ }^{2}$

In lieu of an abstract existence proof, we will attempt to demonstrate the existence of a solution numerically, employing a finite element method. Strictly speaking, this method is only guaranteed to converge for elliptic problems, but we will proceed anyway under the assumption that near-ellipticity is enough. The danger that we face is that along the line $\theta_{1}=\theta_{2}$ where ellipticity fails, everything is a solution, including spurious behavior, and the numerics can potentially be quite unstable.

We have used the publicly available FreeFEM++ package [42] to perform the numerical calculations. This software uses the variational, or "weak" formulation of the finite-element problem. We use the default UMFPACK algorithm. In the calculations shown below, we have used a mesh with 2558 vertices, and we have checked by varying the mesh that the solutions we obtain are not mesh-dependent.

A trick that is useful in taming the spurious solutions of our non-elliptic PDE is to impose Dirichlet boundary conditions whenever possible. Fortunately the asymptotic analysis of the preceding subsections allows us to do precisely this. The boundary conditions we have chosen are that $\beta$ vanishes when either angle $\theta_{i}=\pi$ as in (4.45) and along the boundary where $\theta_{i} \rightarrow 0$ we require that $\beta$ is given by the asymptotic form in (4.65) with $b=k$ as determined by the analysis in section 4.3.4.

\footnotetext{
${ }^{2}$ Incidentally, the fact the we obtained an ordinary differential equation rather than a PDE in the limit of section 4.3 .3 was a sign of this degeneracy. Sometimes it is possible for such a degenerate equation to be rendered elliptic by cleverly dividing by zero, but in this case it is not clear to us that such a technique is applicable, as it would make the asymptotic ODE (4.62) singular.
} 
Although the flux due to $\beta$ is finite, in our formulation the function $\beta$ diverges as $\theta_{1}$ and $\theta_{2}$ simultaneously vanish, so we have solved instead for a rescaled function $\gamma$ defined by

$$
\gamma\left(\theta_{1}, \theta_{2}\right)=\left(1-\cos ^{2} \frac{\theta_{1}}{2} \cos ^{2} \frac{\theta_{2}}{2}\right) \beta\left(\theta_{1}, \theta_{2}\right) .
$$

which does not have this singular behavior. We also impose a small cutoff, $\theta_{i}>\theta_{c}=10^{-7}$ to prevent problems with division by zero. To be specific, our boundary conditions for $\gamma$ take the form

$$
\begin{aligned}
& \gamma\left(\theta_{1}, \pi\right)=\gamma\left(\pi, \theta_{2}\right)=0 \\
& \gamma\left(\theta_{1}, \theta_{c}\right)=-2 k+\frac{2 k}{\sin ^{2} \frac{\theta_{1}}{2}} \frac{\log \sin \frac{\theta_{c}}{2}-\log \sin \frac{\theta_{1}}{2}}{\log \sin \frac{\theta_{c}}{2}+\log \sin \frac{\theta_{1}}{2}} \\
& \gamma\left(\theta_{c}, \theta_{2}\right)=-2 k+\frac{2 k}{\sin ^{2} \frac{\theta_{2}}{2}} \frac{\log \sin \frac{\theta_{c}}{2}-\log \sin \frac{\theta_{2}}{2}}{\log \sin \frac{\theta_{c}}{2}+\log \sin \frac{\theta_{2}}{2}}
\end{aligned}
$$

This form was chosen to agree with the asymptotic solution (4.73) in the limit $\theta_{c} \rightarrow 0$ and to be compatible with the solution (4.65) in the "interpolating" limit.

The result for $\gamma$ is shown in figure 1. We see that the gradient of $\gamma$ appears to grow near the boundaries $\theta_{i} \rightarrow 0$, consistent with the subleading asymptotic dependence $1 / \log \theta_{i}$. Near the origin, this effect becomes more pronounced; we believe it reflects the behavior of the subleading asymptotics in the $\theta_{i}$ coordinates and does not represent a physical singularity. The most important thing to take from this diagram is that the solution exists and it appears to be free of spurious behavior.

For the second function $\alpha$ we have implemented the procedure of constructing a Poisson equation with a $\beta$-dependent source term. The boundary conditions on $\alpha$ are of generalized Neumann type - the normal derivatives of $\alpha$ must be compatible with the first order system of equations (4.42). Strictly speaking, these boundary conditions leave our problem slightly ill-posed, but the ambiguity simply corresponds to shifting $\alpha$ by a constant, which was expected because the basis form $P$ was closed. Figure (2) shows our result for $\alpha$. The mildly singular behavior at the origin appears to be compatible with the log $u$ dependence expected from (4.68), and near the corner $\left(\theta_{1}=\pi, \theta_{2}=\pi\right)$ it appears that $\alpha$ flattens out and approaches a constant.

\subsubsection{Summary of results}

Because the calculation of the supersymmetry-restoring flux was quite complicated, let us pause to summarize the result. The self-dual $(1,1)$ flux can be written as a linear combination of two basis two-forms $P$ and $Q$ which are defined in (4.35). We have constructed a full solution numerically which appears to be smooth everywhere in the interior of the brane worldvolume, and which is presented graphically in figures (1) and (2).

The fact that our solution for the D7-brane flux is numerical is somewhat unfortunate, as it makes the flux awkward to use in calculations. However, the numerical solution does have relatively simple behavior at large and small radius, and we have given analytic expressions for the large radius asymptotics. The leading asymptotics are consistent with 


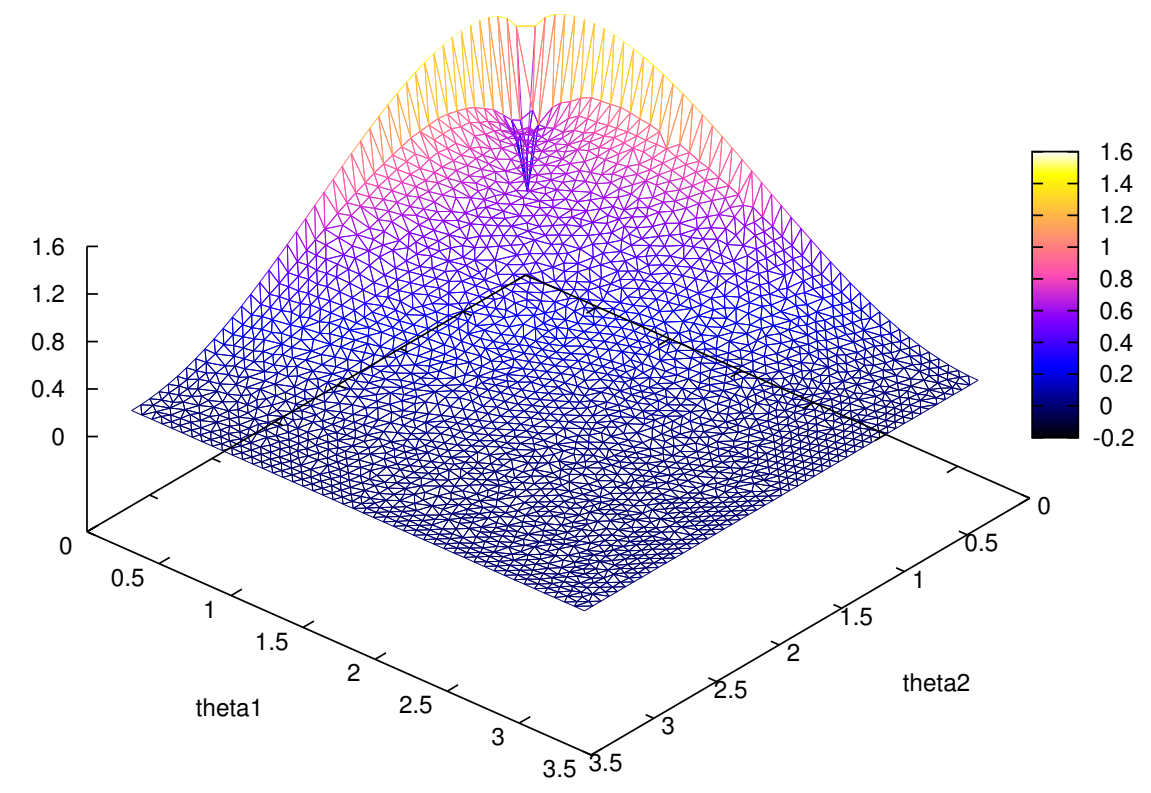

Figure 1. The $\gamma$ component of the flux, as found by a numerical calculation. We have set $k=1$.

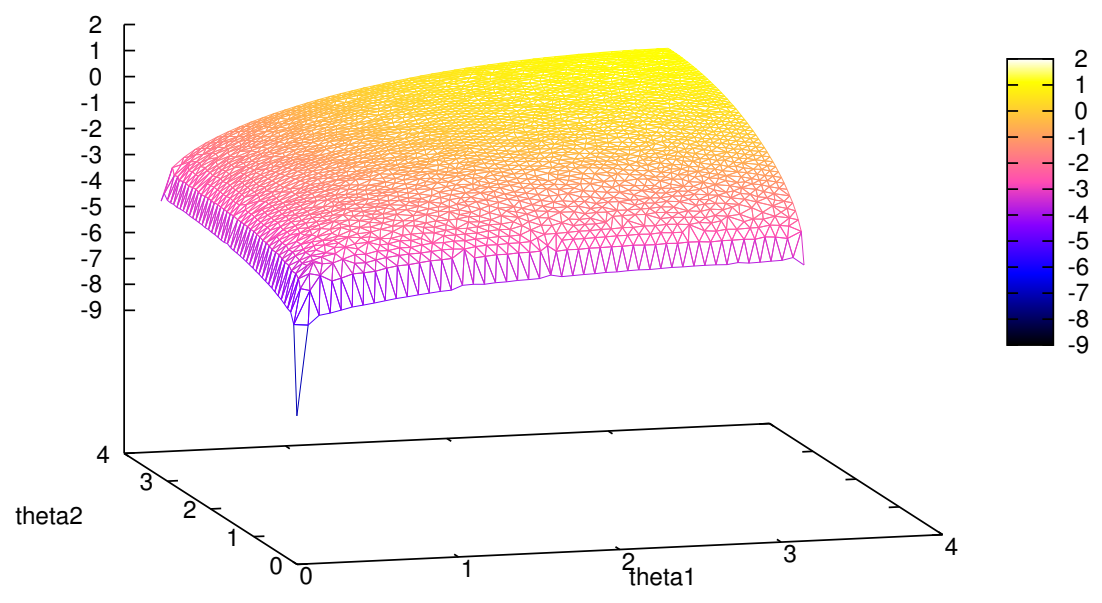

Figure 2. The $\alpha$ component of the flux, computed numerically in terms of the angles $\theta_{i}$. We have set $k=1$.

the proposal of Benini [41]; we have extended his result by demonstrating that the flux can be continued over the entire D7-brane worldvolume.

The small radius asymptotics $\left(\theta_{1} \approx \pi\right.$ and $\theta_{2} \approx \pi$ simultaneously) are that $\beta=0$ and $\alpha$ is a constant. 
When one angle (say $\theta_{2}$ ) is taken to be small, the leading behavior of $\beta$ is

$$
\beta=2 k \cot ^{2} \frac{\theta_{1}}{2}
$$

which is consistent with the earlier proposal of [41] (see appendix D), and $\alpha$ has the leading behavior

$$
\alpha=\alpha_{0}-2 k \log \left|\log \frac{\theta_{2}}{2}\right|
$$

Here the $\log \log$ behavior gives a deviation from the result of [41].

When both angles are small, the asymptotic analysis is somewhat different. In terms of the coordinates defined by (4.54) and (4.55), the leading asymptotic is given by

$$
\beta=k e^{u}\left(\frac{1}{u} \frac{v}{\sinh v}\right)-2 k
$$

where we have included the $-2 k$ at the first subleading order in $e^{-u}$, and the leading asymptotic for $\alpha$ is

$$
\alpha=\alpha_{0}-2 k \log u
$$

\subsection{Other embeddings}

In this subsection we point out that several well-known examples of D7-branes embeddings are not supersymmetric without the inclusion of worldvolume gauge flux. It would be interesting, though presumably quite difficult, to study whether a supersymmetry-restoring flux exists in each case.

Interestingly, given a holomorphic embedding there is a simple criterion that in many cases can immediately show that D7 worldvolume flux is necessary for supersymmetry. When the D7-brane is embedded holomorphically, it is possible to write its embedding equation in terms of the $w_{i}$ coordinates: $g\left(w_{i}\right)=0$, say. Then if $g\left(w_{i}\right)$ is a polynomial, in the limit of large radius $r$ one may approximate $g\left(w_{i}\right)$ by truncating to its terms of highest rank. If the resulting homogeneous polynomial contains a factor of one of the $w_{i}$ (up to an $\mathrm{SO}(4)$ rotation), then in the large radius limit the brane embedding admits a branch which locally looks like the simple $w$-embedding, which as we have already seen requires a nontrivial flux to be supersymmetric.

Let us now turn to some examples that have been studied in the literature. The holomorphic embedding (4.1) admits a generalization that was proposed in [33]

$$
z_{4}=f\left(z_{1}^{2}+z_{2}^{2}\right)
$$

where $f(x)$ is an arbitrary function of $x=z_{1}^{2}+z_{2}^{2}$. However, we may also write $x=2\left(w_{1} w_{2}\right)$, so if $f(x)$ is a polynomial, then in the large radius limit $f \sim\left(w_{1} w_{2}\right)^{p}$ for some $p$, and it may be approximated by $p$ copies of the $w_{1}=0$ and $w_{2}=0$ embeddings. Based on the argument given above, we would expect this embedding to be non-supersymmetric without 
a worldvolume flux, and we have checked this explicitly in appendix B. A similar argument applies to the Karch-Katz embedding [31]:

$$
w_{1} w_{2}=\frac{z_{1}^{2}+z_{2}^{2}}{2}=\mu^{2}, \quad \mu \in \mathbb{C},
$$

and the interested reader will find details of the check in appendix C. Finally, there is an interesting class of embeddings proposed by Arean et al in [43] given by

$$
\prod_{i=1}^{4} w_{i}^{p_{i}}=\mu^{P} .
$$

This embedding admits a factorization in terms of the $w_{i}$ in the large radius limit, so based on the examples we have studied so far we expect that it will require a worldvolume flux to be supersymmetric.

One might hope that, as in the case of the $w$-embedding, that the necessary worldvolume fluxes might be constructible for more general embeddings - the fact that the flux we constructed vanishes for the part of the D7-brane that dips into the throat is a promising sign. The explicit calculations are daunting, however. A general holomorphic embedding will break all the isometries of the conifold, and the resulting system of equations one would need to solve to find the flux can be a system of partial differential equations for three functions of four variables. Ideally one would hope for a more abstract proof of existence for solutions, and we leave this as an interesting but difficult open problem.

\section{$5 \quad$ Field theory remarks}

The D7-branes in the warped conifold geometry that we have studied in the previous section have an interesting interpretation in the dual gauge theory. In this section we make some remarks about the field theories for the various brane embeddings we have studied in the earlier part of the paper (For a good review of cascading field theories, see [44].).

When no D7-branes are present, the dual gauge theory of the warped deformed conifold is a non-conformal field theory with a product gauge group $\mathrm{SU}(N+M) \times \mathrm{SU}(N)$ and matter fields $A_{1,2}, B_{1,2}$ which transform in the bifundamental color representations $(\mathbf{N}+\mathbf{M}, \overline{\mathbf{N}})_{c}$ and $(\overline{\mathbf{N}+\mathbf{M}}, \mathbf{N})_{c}$. The theory also has a superpotential

$$
W=\lambda \operatorname{Tr}\left(A_{i} B_{j} A_{k} B_{l}\right) \epsilon^{i k} \epsilon^{j l} .
$$

When $M=0$, the gauge theory is actually superconformal [45]. Moreover, one can show that the moduli space of the field theory corresponds to the moduli space of $N$ D3 branes probing the conifold geometry. To see this, take some number of D3-branes and move them away from the tip of the conifold by giving expectation values to the fields $A_{i}, B_{j}$. Imposing the F-term and D-term equations, one discovers that with the identifications

$$
w_{1}=A_{1} B_{1}, \quad w_{2}=A_{2} B_{2}, \quad w_{3}=A_{1} B_{2}, \quad w_{4}=A_{2} B_{1} .
$$

we recover precisely the defining equation of the conifold (2.7). 
When $M \neq 0$, conformal invariance is broken and the theory undergoes renormalization group flow $[25,37]$. Along the $\mathrm{RG}$ flow, the two gauge group factors take turns being strongly coupled; when one of them goes to strong coupling, one can perform a Seiberg duality which results in a weakly coupled description. Each Seiberg duality causes the gauge group to change from $\mathrm{SU}(N+M) \times \mathrm{SU}(N)$ to $\mathrm{SU}(N) \times \mathrm{SU}(N-M)$ [46]. Ultimately, in the far IR, this "cascade" must end, as the gauge group ranks cannot be negative. At that point, the theory undergoes confinement, and has a dynamically generated scale which is related to the deformation parameter of the conifold [25].

When we add D7-branes in the gravity theory, the gauge theory is correspondingly modified by the addition of a number of matter fields charged as fundamentals of the gauge group. The introduction of additional matter should change the duality cascade pattern, and it was argued in [34] that the extra matter causes the duality cascade to slow down as the RG scale decreases.

\subsection{Cascade pattern for $z$ embedding and its generalizations}

The analogue of the KS duality cascade when we include probe branes embedded by $z_{1}=\mu$, is actually quite simple, and is manifestly self-similar, as was pointed out in [47]. Although the discussion in this subsection will contain nothing new for experts, for the sake of explicitness, we review here the cascade analysis.

Because the worldvolume flux can vanish for the supersymmetric $z$-embedding, it is reasonable to guess that the appropriate field theory description for the $z$-embedding in the KS background differs from that of the singular conifold theory only by changing the ranks of the gauge groups from $\mathrm{SU}(N) \times \mathrm{SU}(N)$ to $\mathrm{SU}(N+M) \times \mathrm{SU}(N)$. The superpotential for the case of one such D7 brane (which one can motivate based on RG flow from a related orbifold theory [34]) is

$$
W_{z_{1}=\mu}=\lambda_{1} q\left(A_{\alpha} B_{\alpha}-\mu\right) \tilde{q}+\lambda_{2} \frac{(q \tilde{q})^{2}}{2} .
$$

the additional quarks $q$ and $\tilde{q}$ transform in the $(\mathbf{N}+\mathbf{M}, \mathbf{1})$ and $(\overline{\mathbf{N}+\mathbf{M}}, \mathbf{1})$ representations of the $\mathrm{SU}(N+M) \times \mathrm{SU}(N)$ gauge group (of course we can also choose $q$ and $\tilde{q}$ to be charged under the $\mathrm{SU}(N)$ gauge group instead.) One check of this superpotential is that if we probe the theory with a D3-brane, then the quarks, which one can think of as D3-D7 strings, become massless precisely when the probe D3-brane intersects with the D7. The superpotential has an obvious generalization to the case of $K$ coincident D7-branes. Let us consider the case where the additional quark $q$ transforms in the $(\mathbf{N}+\mathbf{M}, \mathbf{1})$ representation, so that the quark is coupled to the $\mathrm{SU}(N+M)$ factor which flows to strong coupling. As in the case of the regular KS cascade, there are mesonic operators $M_{\alpha \beta}=A_{\alpha} B_{\beta}$, and because of the added quarks there are also operators which we label as $C, D$ and $N$ :

$$
\left(C_{\alpha}\right)_{a}^{m}=\left(A_{\alpha}\right)_{i}^{m} \tilde{q}_{a}^{i}, \quad\left(D_{\alpha}\right)_{m}^{a}=q_{i}^{a}\left(B_{\alpha}\right)_{m}^{i}, \quad N_{b}^{a}=q_{i}^{a} \tilde{q}_{b}^{i}, \quad i=1 \ldots N+M, a, b=1, \ldots, K
$$


These additional $\mathrm{SU}(N+M)$-invariant quantities and along with the original mesons $M_{\alpha \beta}$ allow us to rewrite the total modified superpotential for the $z$ embedding into:

$$
W_{\text {Total }}=h \operatorname{Tr}\left(M_{\alpha \beta} M_{\gamma \delta}\right) \epsilon^{\alpha \gamma} \epsilon^{\beta \delta}+\lambda_{1} \operatorname{Tr}\left(D_{\alpha} C_{\alpha}-\mu N\right)+\lambda_{2} \frac{N^{2}}{2} .
$$

Now, as the $\mathrm{SU}(N+M)$ factor flows to strong coupling, we see that we have $N_{c}=N+M$ and $N_{f}=2 N+K$, so we are in the conformal window and may perform a Seiberg duality. Under this duality, the weakly coupled theory with gauge group $\mathrm{SU}(N-(M-K)) \times \mathrm{SU}(N)$, and $2 N+K$ flavors coupled to the $\mathrm{SU}(N-(M-K))$ factor. Notice that in contrast with the original KS theory, where the difference between the ranks of two gauge groups remain unchanged throughout the duality cascade, here the difference between the ranks reduces by $K$ at each step of the cascade. The resulting dual superpotential now receives the following additional contributions:

$$
\frac{1}{\Xi}\left[\operatorname{Tr}\left(M_{\alpha \beta} a^{\alpha} b^{\beta}\right)+\operatorname{Tr}\left(C_{\alpha} q^{\prime} b^{\alpha}\right)+\operatorname{Tr}\left(D_{\alpha} a^{\alpha} \tilde{q}^{\prime}\right)+\operatorname{Tr}\left(N q^{\prime} \tilde{q}^{\prime}\right)\right] .
$$

Here $a_{\alpha}$ and $b_{\alpha}$ are the dual fields for $A_{\alpha}$ and $B_{\alpha}$, under the $\mathrm{SU}(N-(M-K)) \times \mathrm{SU}(N)$ gauge group, they transform as $(\mathbf{N}-(\mathbf{M}-\mathbf{K}), \overline{\mathbf{N}})$ and $(\overline{\mathbf{N}-(\mathbf{M}-\mathbf{K})}, \mathbf{N})$ representations respectively; whereas the $K q^{\prime}$ and $\tilde{q}^{\prime}$ s, the dual quarks and anti-quarks for $q$ and $\tilde{q}$ transforms in the $(\mathbf{N}-(\mathbf{M}-\mathbf{K}), \mathbf{1})$ and $(\overline{\mathbf{N}-(\mathbf{M}-\mathbf{K})}, \mathbf{1})$ representations.

Now, we may integrate out the $\mathrm{SU}(N-(M-K))$ singlets. First, note that one obtains the following F-term equations:

$$
\begin{gathered}
M_{\alpha \beta}: 2 h \epsilon^{\alpha \gamma} \epsilon^{\beta \delta} M_{\gamma \delta}+\frac{1}{\xi} a^{\alpha} b^{\beta}=0, \\
N: \lambda_{2} N-\mu+\frac{1}{\xi} q^{\prime} \tilde{q}^{\prime}=0, \\
C_{\alpha}: \lambda_{1} D_{\alpha}+\frac{1}{\xi} q^{\prime} b_{\alpha}=0 \\
D_{\alpha}: \lambda_{1} C_{\alpha}+\frac{1}{\xi} a_{\alpha} \tilde{q}^{\prime}=0 .
\end{gathered}
$$

Substituting (5.7)-(5.10) into (5.5) and (5.6), we obtain the superpotential for the weakly coupled $\mathrm{SU}(N-(M-K)) \times \mathrm{SU}(N)$ theory:

$$
W_{\text {Dual }}=-\frac{1}{\xi^{2}}\left(\frac{1}{4 h} \operatorname{Tr}\left(a_{\alpha} b_{\beta} a_{\gamma} b_{\delta}\right) \epsilon^{\alpha \gamma} \epsilon^{\beta \delta}+\frac{1}{\lambda_{1}} q^{\prime}\left(b_{\alpha} a_{\alpha}-\frac{\xi \mu}{\lambda_{2}}\right) \tilde{q}^{\prime}+\frac{1}{2 \lambda_{2}}\left(q^{\prime} \tilde{q}^{\prime}\right)^{2}\right) .
$$

This superpotential (5.11) has exactly the same functional form (up to field re-definitions and extra constants) as the original superpotential (5.1) and (5.3), except that the quarks are charged under the gauge group which flows to weak coupling.

Now let us note that if the additional quarks $q, \tilde{q}$ are coupled to the gauge group that flows to weak coupling, the analysis proceeds essentially as before, except that it is simpler because the quarks $q$ and $\tilde{q}$ do not bind to form mesons. After performing a Seiberg duality on the strongly coupled gauge group, the two gauge groups switch roles again and the quarks $q, \tilde{q}$ will again be coupled to the gauge group which flows to strong coupling. Thus after two steps of the cascade, the theory is fully self-similar. 


\subsection{The vacua for Kuperstein-like embeddings}

As was shown in [33] and reviewed in section 4.1, the simple $z$-embedding is supersymmetric for all $\mu$ on the full deformed conifold. Therefore it is interesting to study the case of a D7-brane embedded by $z_{1}=\mu$ in the extreme infrared $(\mu \sim \epsilon)$, where the conifold becomes deformed. In this case, the quarks due to the D7-brane have masses of the order of the confinement scale and can modify the IR dynamics of the theory.

Suppose the number of units of 3-form flux, after having decreased from the D7 backreaction, is $M^{* 3}$. To investigate the moduli space of the theory, we use the standard trick of probing the theory by adding a single mobile D3-brane. The gauge group on the D3 brane probe is $\mathrm{SU}\left(M^{*}+1\right) \times \mathrm{U}(1)$, and we have matter fields $a_{\alpha}$ and $b_{\alpha}$ transforming respectively in $\left(\mathbf{M}^{*}+\mathbf{1}, \mathbf{1}\right)$ and $\left(\mathbf{1}, \mathbf{M}^{*}+\mathbf{1}\right)$ representations, moreover we also have an additional quark $q^{\prime}$ coming from 3-7 string transform in $\left(\mathbf{M}^{*}+\mathbf{1}, \mathbf{1}\right)$. By self-similarity, the UV superpotential on the probe here is essentially identical to (5.11), but with the fields and the parameters modified accordingly. At low energy, the UV superpotential can be written again in terms of the $\mathrm{SU}\left(M^{*}+1\right)$ invariant quantities and it also acquires a non-perturbative Affleck-Dine-Seiberg superpotential [48]:

$$
W_{\mathrm{ADS}}=\left(M^{*}-2\right)\left(\frac{\tilde{\Lambda}^{3 M^{*}}}{\operatorname{det} \Omega}\right)^{\frac{1}{M^{*}-2}} .
$$

Here the determinant of the $3 \times 3$ meson matrix $\operatorname{det} \Omega$ is given by

$$
\operatorname{det} \Omega=\frac{N}{2} \epsilon^{\alpha \gamma} \epsilon^{\beta \delta} M_{\alpha \beta} M_{\gamma \delta}-\epsilon^{\alpha \gamma} \epsilon^{\beta \delta} C_{\alpha} D_{\beta} M_{\gamma \delta},
$$

whereas the various gauge invariant quantities are defined similarly as in (5.4). From (5.12) and (5.13) we can deduce the $F$-term equations:

$$
\begin{aligned}
M_{\alpha \beta} & : \epsilon^{\alpha \gamma} \epsilon^{\beta \delta}\left(h M_{\alpha \beta}-S(\Omega)\left(N M_{\alpha \beta}-C_{\alpha} D_{\beta}\right)\right)=0, \\
N & : \lambda_{2} N+\frac{1}{2} S(\Omega) \epsilon^{\alpha \gamma} \epsilon^{\beta \delta} M_{\alpha \beta} M_{\gamma \delta}=\lambda_{1} \mu, \\
C_{\alpha} & : \lambda_{1} \epsilon^{\alpha \beta} D_{\beta}=S(\Omega) \epsilon^{\alpha \gamma} \epsilon^{\beta \delta} D_{\beta} M_{\gamma \delta}, \\
D_{\alpha} & : \lambda_{1} \epsilon^{\alpha \beta} C_{\alpha}=S(\Omega) \epsilon^{\alpha \gamma} \epsilon^{\beta \delta} C_{\alpha} M_{\gamma \delta} .
\end{aligned}
$$

with

$$
S(\Omega)=\left(\frac{\tilde{\Lambda}^{3 M^{*}}}{\operatorname{det} \Omega}\right)^{\frac{1}{M^{*}-2}} \frac{1}{\operatorname{det} \Omega} .
$$

One class of simple solutions to these F-term equations is given by setting $C_{\alpha}=D_{\alpha}=0$ and we have

$$
\begin{aligned}
N & =\frac{1}{2 \lambda_{2}}\left(\lambda_{1} \mu \pm \sqrt{\left(\lambda_{1} \mu\right)^{2}-4 \lambda_{2} h \operatorname{det} M_{\alpha \beta}}\right), \\
h \operatorname{det} M_{\alpha \beta} & =\left(\frac{\tilde{\Lambda}^{3 M^{*}}}{N \operatorname{det} M_{\alpha \beta}}\right)^{\frac{1}{M^{*}-2}} .
\end{aligned}
$$

\footnotetext{
${ }^{3}$ The explicit value $M^{*}$ can be deduced from the pattern of the flavor cascade described earlier to be $M^{*}=\sqrt{(M-K / 2)^{2}-2 N K}-K / 2$.
} 
The deformed conifold branch naturally appears when we consider the limit $\left(\lambda_{1} \mu\right)^{2} \ll$ $4 \lambda_{2} h \operatorname{det} M_{\alpha \beta}$, which yields:

$$
\operatorname{det} M_{\alpha \beta} \approx\left(\frac{\lambda_{2} \tilde{\Lambda}^{6 M^{*}}}{h^{2 M^{*}-3}}\right)^{\frac{1}{2 M^{*}-1}}
$$

Another extreme limit which can be taken is that $\left(\lambda_{1} \mu\right)^{2} \gg 4 \lambda_{2} h \operatorname{det} M_{\alpha \beta}$, and again simple algebra shows that we recover the deformed conifold. These limits precisely correspond to moving the probe D3-brane away from the flavor D7 branes, and locally it detects the deformed conifold geometry.

It would be very interesting to understand the other regions of the moduli space especially with $C_{\alpha}, D_{\beta} \neq 0$.

\section{$5.3 w$-embedding and its generalizations}

Now let us move to the cascade analysis for $w$-embedding and its generalizations, such as the ACR embeddings [43]. We shall begin by relating the superpotential for various ACRembeddings to the one for the simplest $w$-embedding (4.10); we then on the cascade pattern for the simplest $w$-embedding, as the more complicated cases can be analyzed similarly.

Recall that for the $w$-embedding (4.10), an appropriate additional term to the KS superpotential (5.1) was given in [34]:

$$
W_{w_{1}=\mu}=\lambda q\left(A_{1} B_{1}-\mu\right) \tilde{q}
$$

where $q$ can transforms in the representation $(\mathbf{N}+\mathbf{M}, \mathbf{1})$ and $\tilde{q}$ transforms in the $(\overline{\mathbf{N}+\mathbf{M}}, \mathbf{1})$ representation of the gauge group. This superpotential can be generalized to many other interesting cases. Take for example the Karch-Katz embedding [31], $w_{1} w_{2}=\mu$. In the large radius limit, we may approximate this embedding equation by $w_{1} w_{2}=0$, which factorizes into two branches $w_{1}=0$ and $w_{2}=0$. On the field theory side, this naturally corresponds to introducing two sets of additional quarks $\left\{q_{1}\right\}$ and $\left\{q_{2}\right\}$ with the superpotential

$$
\kappa_{1} q_{1}\left(A_{1} B_{1}\right) \tilde{q}_{1}+\kappa_{2} q_{2}\left(A_{2} B_{2}\right) \tilde{q}_{2}
$$

At smaller radius, however, the two branches meet and at least one set of quarks will be massive. We can describe this with superpotential terms of the form

$$
\gamma_{1} q_{2} \tilde{q}_{1}+\gamma_{2} q_{1} \tilde{q}_{2} \text { with } \gamma_{1} \gg \gamma_{2}
$$

Taking the larger of the two masses and integrating out the associated quarks, we obtain a superpotential of the form $q\left[\left(A_{1} B_{1}\right)\left(A_{2} B_{2}\right)+\gamma_{2}\right] \tilde{q}$, as desired. Of course if we continue further and integrate out quarks using the $\gamma_{2}$ mass term we will be left with a trivial superpotential.

Now, for the more general ACR embedding, we can implement a generalization of the procedure we have described for the Karch-Katz embedding. Again, the ACR equation 
can be factorized into different branches in the vanishing $\mu$ limit, each of which is given by $w_{i}=0$ for some $i$, motivating superpotential terms of the form

$$
\begin{aligned}
\sum_{n_{1}=1}^{p_{1}} \kappa_{n_{1}} q_{n_{1}}\left(A_{1} B_{1}\right) \tilde{q}_{n_{1}}+\sum_{n_{2}=1}^{p_{2}} \kappa_{n_{2}} q_{n_{2}}\left(A_{2} B_{2}\right) \tilde{q}_{n_{2}} \\
+\sum_{n_{3}=1}^{p_{3}} \kappa_{n_{3}} q_{n_{3}}\left(A_{1} B_{2}\right) \tilde{q}_{n_{3}}+\sum_{n_{4}=1}^{p_{4}} \kappa_{n_{4}} q_{n_{4}}\left(A_{2} B_{1}\right) \tilde{q}_{n_{4}},
\end{aligned}
$$

which are simply linear combinations of the simplest $w$-embedding. By adding appropriate mass terms and integrating out quarks as they become massive, it is not hard to see that we can flow to a superpotential of the form

$$
W_{\mathrm{ACR}}=\lambda q\left[\left(A_{1} B_{1}\right)^{p_{1}}\left(A_{2} B_{2}\right)^{p_{2}}\left(A_{1} B_{2}\right)^{p_{3}}\left(A_{2} B_{1}\right)^{p_{4}}-\mu^{P}\right] \tilde{q}
$$

which is related to the ACR embedding equation in a direct way.

Now, in the case with no three-form flux on the conifold, the D7 branes in the $w$ embedding factorizes further into two branches, using the relation (5.2) $w_{1}=A_{1} B_{1}$. A superpotential reflecting this factorization property was proposed in [34] and studied in [41]:

$$
W_{\text {flav }}=g_{1} Q A_{1} \tilde{q}+g_{2} q B_{1} \tilde{Q} .
$$

By introducing the quark mass terms $m_{1} q \tilde{q}+m_{2} Q \tilde{Q}, m_{2} \gg m_{1}$ the familiar superpotential term (5.21) (up to appropriate redefinition) can be recovered by introducing the quark mass terms $m_{1} q \tilde{q}+m_{2} Q \tilde{Q}$ and integrating out appropriately. The cascade pattern for this type of superpotential has been studied in [41], with the result that the self-similarity under Seiberg duality transformation no longer presents in this case and a sequence of additional terms involving singlet states and quarks is generated. Explicitly the resulting superpotential after a step of cascade is given by:

$$
\lambda_{1} \operatorname{Tr}\left(a_{\alpha} b_{\beta} a_{\gamma} b_{\delta}\right) \epsilon^{\alpha \gamma} \epsilon^{\beta \delta}+\lambda_{2}\left(\bar{P} a_{2} q^{\prime}+\tilde{q}^{\prime} b_{2} P+\Sigma_{0} q^{\prime} \tilde{q}^{\prime}\right) .
$$

Here $\lambda_{1,2}$ are combinations of coupling constants and dynamical scale; $\Sigma_{0}$ is an additional gauge singlet field. In the proposal of [41], the cascade is almost self-similar; at each step the term with the singlet $\Sigma$ acquires additional $A, B$ fields and (at least naively) becomes more and more irrelevant. This unusual cascade deserves further study; perhaps the worldvolume flux we constructed in section 4.3 may shed some light on the nature of the field theory.

\section{Discussion}

We conclude with a few comments on extensions and possible applications of our results. Having seen that worldvolume fluxes can restore supersymmetry, it would of course be interesting to study fluxes for embeddings other than the simple linear $w$-embedding, and to extend our calculations to the deformed conifold. As we have already remarked, this is in general a daunting mathematical exercise, but perhaps it will be possible to prove an 
existence theorem of some kind for the necessary fluxes, possibly based on the topology of the embedding. Understanding the worldvolume flux may also help in extending the calculations of D7-brane backreaction along the lines of [47, 49]. It would also be interesting to study D7-branes in warped cones other than the conifold.

In the gauge/gravity duality, these D7-branes add flavor to the dual KS gauge theory, with corresponding prospects for understanding mesons and baryons in a confining theory. It might be interesting to revisit the calculations of the meson spectrum, as for example in [50], with worldvolume fluxes turned on. In particular, it would be interesting to understand better the physical meaning of the closed $(1,1)$ form $P$ which we identified in section 4.3, which one can turn on without breaking any supersymmetry; it should correspond to deformation of the field theory by some operator. A clearer understanding of these supersymmetry-restoring fluxes may also be important in clarifying the nature of the duality cascade with probe $w$-D7-branes.

There are also potential applications to inflationary model building. The model constructed in [28] used a very special brane embedding to generate the inflationary potential, but with several different supersymmetric brane embeddings, one can imagine combining D7-branes in various ways to obtain many other inflationary models. It would also be very interesting to understand if the presence of extra flux can modify the explicit expression for the gaugino condensate given in [27]. It was argued in [28] that at the leading order expansion of D7 DBI action, the additional supersymmetric flux should not modify the results obtained in [27]. Combining the severe constraints of holomorphy of the superpotential and the global symmetries preserved by the embedding, one is tempted to conclude that the potential due to the gaugino condensate in the deformed conifold should be quite similar to that of the singular conifold. Having a supersymmetric D7 configuration including the necessary world volume flux should allow us check such a statement explicitly, following the calculations in [27].

Also, it would also be interesting to consider small deviations from the extremal limit, and allow supersymmetry to be softly broken by a D-term potential whose magnitude is set by $\int_{\Sigma_{4}} J \wedge \mathcal{F}$ where $\Sigma_{4}$ is the four cycle which D7 brane wraps on in the deformed conifold [51] (see also [52] for an interesting construction in the resolved conifold.) Combined with the fact that $\Sigma_{4}$ is generally warped, this may allow us to consider a variety of interesting scenarios for supersymmetry breaking.

\section{Acknowledgments}

It is a pleasure to thank Francesco Benini, Anatoly Dymarsky, Mikhail Feldman, Fernando Marchesano, Bret Underwood, and Angel Uranga for helpful discussions. We also thank an anonymous referee for very useful criticisms. HYC would like to thank KITP at UCSB, the Sixth Simons Workshop at SUNY Stony Brook and Institute for Advanced Study, Princeton for their hospitality while part of the work was being carried out. This work was supported in part by NSF CAREER Award No. PHY-0348093, DOE grant DE-FG02-95ER40896, a Research Innovation Award and a Cottrell Scholar Award from Research Corporation, and a Vilas Associate Award from the University of Wisconsin. 


\section{A Deformed conifold coordinates}

The embedding coordinates $\left\{z_{i}\right\}$ transform as $\mathbf{4}$ under the $\mathrm{SO}(4)$ isometry group. An alternative parametrization for the deformed conifold constraint (2.12) can also be given by a $2 \times 2$ complex matrix:

$$
W=\frac{1}{\sqrt{2}}\left(\begin{array}{cc}
z_{3}+i z_{4} & z_{1}-i z_{2} \\
z_{1}+i z_{2} & -z_{3}+i z_{4}
\end{array}\right) \equiv\left(\begin{array}{cc}
w_{3} & w_{2} \\
w_{1} & w_{4}
\end{array}\right),
$$

defining equation for the conifold can then be expressed in terms of $W$ as

$$
\operatorname{det} W=w_{3} w_{4}-w_{1} w_{2}=-\frac{\epsilon^{2}}{2} .
$$

The coordinates $\left\{w_{i}\right\}$ now transform as $(\mathbf{2}, \mathbf{2})$ under $\mathrm{SO}(4) \sim \mathrm{SU}(2)_{L} \times \mathrm{SU}(2)_{R}$ isometry group. The complex embedding coordinates of deformed conifold $\left\{z_{1}, z_{2}, z_{3}, z_{4}\right\}$ can be expressed in terms of the real coordinates $\left\{\rho \in \mathbb{R}, \psi \in[0,4 \pi], \theta_{1,2} \in[0, \pi], \phi_{1,2} \in[0,2 \pi]\right\}$, $S=\rho+i \psi$ as:

$$
\begin{array}{r}
z_{1}=\epsilon\left[\cosh \left(\frac{S}{2}\right) \cos \left(\frac{\theta_{1}+\theta_{2}}{2}\right) \cos \left(\frac{\phi_{1}+\phi_{2}}{2}\right)\right. \\
\left.+i \sinh \left(\frac{S}{2}\right) \cos \left(\frac{\theta_{1}-\theta_{2}}{2}\right) \sin \left(\frac{\phi_{1}+\phi_{2}}{2}\right)\right], \\
z_{2}=\epsilon\left[-\cosh \left(\frac{S}{2}\right) \cos \left(\frac{\theta_{1}+\theta_{2}}{2}\right) \sin \left(\frac{\phi_{1}+\phi_{2}}{2}\right)\right. \\
\left.+i \sinh \left(\frac{S}{2}\right) \cos \left(\frac{\theta_{1}-\theta_{2}}{2}\right) \cos \left(\frac{\phi_{1}+\phi_{2}}{2}\right)\right], \\
z_{3}=\epsilon\left[-\cosh \left(\frac{S}{2}\right) \sin \left(\frac{\theta_{1}+\theta_{2}}{2}\right) \cos \left(\frac{\phi_{1}-\phi_{2}}{2}\right)\right. \\
\left.\quad+i \sinh \left(\frac{S}{2}\right) \sin \left(\frac{\theta_{1}-\theta_{2}}{2}\right) \sin \left(\frac{\phi_{1}-\phi_{2}}{2}\right)\right], \\
z_{4}=\epsilon\left[-\cosh \left(\frac{S}{2}\right) \sin \left(\frac{\theta_{1}+\theta_{2}}{2}\right) \sin \left(\frac{\phi_{1}-\phi_{2}}{2}\right)\right. \\
\left.\quad-i \sinh \left(\frac{S}{2}\right) \sin \left(\frac{\theta_{1}-\theta_{2}}{2}\right) \cos \left(\frac{\phi_{1}-\phi_{2}}{2}\right)\right] .
\end{array}
$$

In the large radius limit $\rho \rightarrow \infty, \epsilon \cosh (\rho / 2) \approx \epsilon \sinh (\rho / 2) \approx \frac{r^{3 / 2}}{\sqrt{2}}$, the deformed conifold coordinates (A.3) and (A.1) smoothly reduce to those of singular conifold.

\section{B Generalized $z$-embeddings}

We next examine a generalization of the holomorphic embedding (4.1) proposed in [33]

$$
z_{4}=f\left(z_{1}^{2}+z_{2}^{2}\right)
$$


where $f(x)$ is an arbitrary function of $x=z_{1}^{2}+z_{2}^{2}=2\left(w_{1} w_{2}\right)$. This class of embedding generally breaks the $\mathrm{SO}(4)$ isometry group down to the $\mathrm{U}(1)$ which rotates $w_{1}$ and $w_{2}$ by opposite phases.

The general expression for the pull-back of NS-NS B-field in this case is given by:

$$
\hat{\eta}_{1}=\left.\eta_{4}\right|_{z_{4}=f(x)}=B_{1 \overline{1}} d z_{1} \wedge d \bar{z}_{1}+B_{1 \overline{2}} d z_{1} \wedge d \bar{z}_{2}+B_{2 \overline{1}} d z_{2} \wedge d \bar{z}_{1}+B_{2 \overline{2}} d z_{2} \wedge d \bar{z}_{2},
$$

with the various components given by the expression in (4.2):

$$
\begin{aligned}
& B_{1 \overline{1}}=2\left\{\left(z_{3} \bar{z}_{2}-z_{2} \bar{z}_{3}\right)\left(\bar{f}^{\prime} \bar{z}_{1}-f^{\prime} z_{1}\right)+\left(f_{2} z_{1}-\bar{f}_{2} \bar{z}_{1}\right)\left(z_{4} \bar{z}_{2}-z_{2} \bar{z}_{4}\right)\right\}, \\
& B_{2 \overline{2}}=-2\left\{\left(z_{3} \bar{z}_{1}-z_{1} \bar{z}_{3}\right)\left(\bar{f}^{\prime} \bar{z}_{2}-f^{\prime} z_{2}\right)+\left(f_{2} z_{2}-\bar{f}_{2} \bar{z}_{2}\right)\left(z_{4} \bar{z}_{1}-z_{1} \bar{z}_{4}\right)\right\}, \\
& B_{1 \overline{2}}=\left(z_{4} \bar{z}_{3}-z_{3} \bar{z}_{4}\right)+2\left\{\left(z_{1}\left(z_{3} \bar{z}_{1}-z_{1} \bar{z}_{3}\right) f^{\prime}+\bar{z}_{2}\left(z_{3} \bar{z}_{2}-z_{2} \bar{z}_{3}\right) \bar{f}^{\prime}\right)-\left(z_{3} \rightarrow z_{4}, f^{\prime} \rightarrow f_{2}\right)\right\}, \\
& B_{2 \overline{1}}=\left(z_{3} \bar{z}_{4}-z_{4} \bar{z}_{3}\right)-2\left\{\left(\bar{z}_{1}\left(z_{3} \bar{z}_{1}-z_{1} \bar{z}_{3}\right) \bar{f}^{\prime}+z_{2}\left(z_{3} \bar{z}_{2}-z_{2} \bar{z}_{3}\right) f^{\prime}\right)-\left(z_{3} \rightarrow z_{4}, f^{\prime} \rightarrow f_{2}\right)\right\} .
\end{aligned}
$$

Here

$$
z_{3}=\sqrt{\epsilon^{2}-\left(x+f^{2}(x)\right)}, \quad f^{\prime}(x)=\frac{d f(x)}{d x} \quad f_{2}=-\frac{1+f^{\prime}(x)}{2 z_{3}},
$$

the expression for the previous case (4.1) is recovered by setting $f^{\prime}(x)=0$. The pull-back of the Kähler two-form for the generalized Kuperstein embedding can be written as:

$$
\begin{aligned}
& \hat{\eta}_{5}=\left.\eta_{5}\right|_{\left(z_{4}=f(x)\right)}=J_{1 \overline{1}}^{\prime} d z_{1} \wedge d \bar{z}_{1}+J_{1 \overline{2}}^{\prime} d z_{1} \wedge d \bar{z}_{2}+J_{2 \overline{1}}^{\prime} d z_{2} \wedge d \bar{z}_{1}+J_{2 \overline{2}}^{\prime} d z_{2} \wedge d \bar{z}_{2} \\
& \hat{\eta}_{4}=\left.\eta_{4}\right|_{\left(z_{4}=f(x)\right)}=J_{1 \overline{1}}^{\prime \prime} d z_{1} \wedge d \bar{z}_{1}+J_{1 \overline{2}}^{\prime \prime} d z_{1} \wedge d \bar{z}_{2}+J_{2 \overline{1}}^{\prime \prime} d z_{2} \wedge d \bar{z}_{1}+J_{2 \overline{2}}^{\prime \prime} d z_{2} \wedge d \bar{z}_{2} .
\end{aligned}
$$

with the components

$$
\begin{array}{rlrl}
J_{1 \overline{1}}^{\prime}=1+\left|z_{1}\right|^{2} p(x), & J_{2 \overline{2}}^{\prime} & =1+\left|z_{2}\right|^{2} p(x), \quad J_{1 \overline{2}}^{\prime}=z_{1} \bar{z}_{2} p(x), & J_{2 \overline{1}}^{\prime}=z_{2} \bar{z}_{1} p(x), \\
J_{1 \overline{1}}^{\prime \prime}=S_{1} \bar{S}_{1}, & J_{1 \overline{2}}^{\prime \prime}=S_{1} \bar{S}_{2}, & S_{2 \overline{1}} \bar{S}_{2}, \\
p(x) & =4\left|f_{2}^{\prime}(x)\right|^{2}+\frac{\left|1+2 f_{1}(x)\right|^{2}}{\left|z_{3}\right|^{2}}, \\
S_{1} & =\frac{1}{z_{3}}\left[\left(z_{3} \bar{z}_{1}-z_{1} \bar{z}_{3}\right)+2 z_{1}\left(z_{3} \bar{z}_{4}-\bar{z}_{3}\right) f^{\prime}(x)\right] \\
S_{2} & =\frac{1}{z_{3}}\left[\left(z_{3} \bar{z}_{2}-z_{2} \bar{z}_{3}\right)+2 z_{2}\left(z_{3} \bar{z}_{4}-\bar{z}_{3}\right) f^{\prime}(x)\right]
\end{array}
$$

Notice here that these expressions reduces to the ones for simple Kuperstein embedding (4.1) when $f^{\prime}(x)=0$. To check the SUSY condition for the generalized embedding (4.87), we can first calculate

$$
\begin{aligned}
\hat{\eta}_{5} \wedge \hat{\eta}_{1}= & \frac{\left(z_{1} \bar{z}_{2}-z_{2} \bar{z}_{1}\right)}{\left|z_{3}\right|^{2}}\left(\left[\bar{z}_{3}\left(1+z_{3}^{2}\right) f^{\prime}(x)-z_{3}\left(1+\bar{z}_{3}^{2}\right) \bar{f}^{\prime}(x)\right]\right. \\
& \left.+\left(z_{3} \bar{z}_{4}-z_{4} \bar{z}_{3}\right)\left[2\left|f^{\prime}(x)\right|^{2}\left(1+\left|z_{3}\right|^{2}\right)+f^{\prime}(x)+\bar{f}^{\prime}(x)\right]\right) d \Omega,
\end{aligned}
$$

where $d \Omega=d z_{1} \wedge d z_{2} \wedge d \bar{z}_{1} \wedge d \bar{z}_{2}$. For an arbitrary $f(x)$, the above expression does not vanish unless $f^{\prime}(x)=0$. One can also in principle calculate $\hat{\eta}_{4} \wedge \hat{\eta}_{1}$, and demonstrate that 
it is non-vanishing for arbitrary $f(x)$. In fact, one can consider a special case $f(x)=x$, i.e. $z_{4}=z_{1}^{2}+z_{2}^{2}$; for each specific value of $z_{4}$, this is identical to Karch-Katz embedding (4.88), which we will also show momentarily to be non-supersymmetric. We thus conclude that the embedding (4.87) cannot be supersymmetric in the deformed conifold for arbitrary function $f(x)$. However, with additional world volume flux, the supersymmetric condition can still in principle be satisfied.

Let us remark also on a statement in [33] that the $\kappa$-symmetry conditions must be satisfied because of a symmetry argument. The argument used the following logic. Under the interchange $z_{1} \rightarrow z_{2}, z_{2} \rightarrow z_{1}$, the Kahler form $J$ and the embedding equation are invariant, while $B_{2}$ gets a minus sign. Thus $\hat{J} \wedge \hat{B}$ acquires a minus sign under the interchange. There is also a rotational symmetry (from the $\mathrm{SO}(3)$ preserved by the embedding equation) rotating $z_{1}$ and $z_{2}$, under which $J, B$, and the embedding equation are all invariant. One can write $\hat{J} \wedge \hat{B}=\phi\left(z_{1}, \overline{z_{1}}, z_{2}, \overline{z_{2}}\right) d z_{1} \wedge d z_{2} \wedge d \bar{z}_{1} \wedge d \bar{z}_{2}$. Then $\phi$ is invariant under the rotation but acquires a minus sign on the interchange, and [33] claimed that the only function which could satisfy both properties is $\phi=0$. This claim is not correct, as $z_{1} \bar{z}_{2}-z_{2} \bar{z}_{1}$ is a counterexample.

\section{Karch-Katz embedding}

We consider next the holomorphic embedding given in [31]

$$
w_{1} w_{2}=\frac{z_{1}^{2}+z_{2}^{2}}{2}=\mu^{2}, \quad \mu \in \mathbb{C},
$$

which explicitly breaks the $\mathrm{SO}(4)$ isometry group into $\mathrm{SO}(2) \times \mathrm{SO}(2)$, and these $\mathrm{SO}(2)$ subgroups act by rotating the phases of the ratios $w_{1} / w_{2}$ and $w_{3} / w_{4}$. In the asymptotic limit $r^{3} \gg|\mu|^{2}$ limit, the embedding (4.88) can in fact be viewed as two copies of the D7 brane $w$-embedding we have earlier (4.10) $w_{1}=0$ and $w_{2}=0$, and they intersect and fuse together at some finite radius by turning on D7-D7 interactions.

The pull-back of the NS-NS two form field in this case is given by calculating:

$$
\hat{\eta}_{1}=\left.\eta_{1}\right|_{\left(w_{1} w_{2}=\mu^{2}\right)}=B_{1 \overline{1}} d z_{1} \wedge d \bar{z}_{1}+B_{1 \overline{3}} d z_{1} \wedge d \bar{z}_{3}+B_{3 \overline{1}} d z_{3} \wedge d \bar{z}_{1}+B_{3 \overline{3}} d z_{3} \wedge d \bar{z}_{3}
$$

where the various components are given by:

$$
\begin{aligned}
& B_{1 \overline{1}}=\left|z_{2}\right|^{-2}\left(z_{1} \bar{z}_{2}-z_{2} \bar{z}_{1}\right)\left(z_{3} \bar{z}_{4}-z_{3} \bar{z}_{4}\right), \\
& B_{1 \overline{3}}=-\left\{\left(z_{2} \bar{z}_{4}-z_{4} \bar{z}_{2}\right)+\left(z_{1} \bar{z}_{4}-z_{4} \bar{z}_{1}\right) \frac{z_{1}}{z_{2}}+\left(z_{2} \bar{z}_{3}-z_{3} \bar{z}_{2}\right) \frac{\bar{z}_{3}}{\bar{z}_{4}}+\left(z_{1} \bar{z}_{3}-z_{3} \bar{z}_{1}\right) \frac{z_{1} \bar{z}_{3}}{z_{2} \bar{z}_{4}}\right\}, \\
& B_{3 \overline{1}}=\left\{\left(z_{2} \bar{z}_{4}-z_{4} \bar{z}_{2}\right)+\left(z_{1} \bar{z}_{4}-z_{4} \bar{z}_{1}\right) \frac{\bar{z}_{1}}{\bar{z}_{2}}+\left(z_{2} \bar{z}_{3}-z_{3} \bar{z}_{2}\right) \frac{z_{3}}{z_{4}}+\left(z_{1} \bar{z}_{3}-z_{3} \bar{z}_{1}\right) \frac{\bar{z}_{1} z_{3}}{\bar{z}_{2} z_{4}}\right\}, \\
& B_{3 \overline{3}}=\left|z_{4}\right|^{-2}\left(z_{1} \bar{z}_{2}-z_{2} \bar{z}_{1}\right)\left(z_{3} \bar{z}_{4}-z_{3} \bar{z}_{4}\right),
\end{aligned}
$$

with

$$
z_{2}^{2}=2 \mu^{2}-z_{1}^{2}, \quad z_{4}^{2}=\left(\epsilon^{2}-2 \mu^{2}\right)-z_{3}^{2} .
$$

The components for the pull-back of the Kähler form $J$ can also be calculated from:

$$
\begin{aligned}
& \hat{\eta}_{5}=\left.\eta_{5}\right|_{\left(w_{1} w_{2}=\mu^{2}\right)}=J_{1 \overline{1}}^{\prime} d z_{1} \wedge d \bar{z}_{1}+J_{3 \overline{3}}^{\prime} d z_{3} \wedge d \bar{z}_{3} \\
& \hat{\eta}_{4}=\left.\eta_{4}\right|_{\left(w_{1} w_{2}=\mu^{2}\right)}=J_{1 \overline{1}}^{\prime \prime} d z_{1} \wedge d \bar{z}_{1}+J_{1 \overline{3}}^{\prime \prime} d z_{1} \wedge d \bar{z}_{3}+J_{3 \overline{1}}^{\prime \prime} d z_{3} \wedge d \bar{z}_{1}+J_{3 \overline{3}}^{\prime \prime} d z_{3} \wedge d \bar{z}_{3}
\end{aligned}
$$


The various functions here are:

$$
\begin{array}{ll}
J_{1 \overline{1}}^{\prime}=1+\frac{\left|z_{1}\right|^{2}}{\left|z_{2}\right|^{2}}, & J_{3 \overline{3}}^{\prime}=1+\frac{\left|z_{3}\right|^{2}}{\left|z_{4}\right|^{2}}, \quad J_{1 \overline{3}}^{\prime}=J_{\overline{3} 1}^{\prime}=0 . \\
J_{1 \overline{1}}^{\prime \prime}=-\frac{\left(z_{1} \bar{z}_{2}-\bar{z}_{1} z_{2}\right)^{2}}{\left|z_{2}\right|^{2}}, & J_{3 \overline{3}}^{\prime \prime}=-\frac{\left(z_{3} \bar{z}_{4}-\bar{z}_{3} z_{4}\right)^{2}}{\left|z_{4}\right|^{2}} \\
J_{1 \overline{3}}^{\prime \prime}=-\frac{\left(z_{1} \bar{z}_{2}-\bar{z}_{1} z_{2}\right)\left(z_{3} \bar{z}_{4}-\bar{z}_{3} z_{4}\right)}{z_{2} \bar{z}_{4}}, & J_{3 \overline{1}}^{\prime \prime}=-\frac{\left(z_{1} \bar{z}_{2}-\bar{z}_{1} z_{2}\right)\left(z_{3} \bar{z}_{4}-\bar{z}_{3} z_{4}\right)}{z_{4} \bar{z}_{2}} .
\end{array}
$$

The SUSY condition (3.5) can be checked by calculating the wedge products in turns:

$$
\hat{\eta}_{5} \wedge \hat{\eta}_{1}=-\frac{\left(z_{1} \bar{z}_{2}-z_{2} \bar{z}_{1}\right)\left(z_{3} \bar{z}_{4}-z_{4} \bar{z}_{3}\right)}{\left|z_{2}\right|^{2}\left|z_{4}\right|^{2}}\left(\left|z_{1}\right|^{2}+\left|z_{2}\right|^{2}+\left|z_{3}\right|^{2}+\left|z_{4}\right|^{2}\right) d \Omega
$$

where $d \Omega=d z_{1} \wedge d z_{3} \wedge d \bar{z}_{1} \wedge d \bar{z}_{3}$. In (C.11) we have a product of positive definite sum with a generically non-vanishing number $-4 \operatorname{Im}\left(z_{1} \bar{z}_{2}\right) \operatorname{Im}\left(z_{3} \bar{z}_{4}\right)$. Similarly we calculate $\hat{\eta}_{4} \wedge \hat{\eta}_{1}$ and obtain:

$$
\hat{\eta}_{4} \wedge \hat{\eta}_{1}=\frac{\left(z_{1} \bar{z}_{2}-z_{2} \bar{z}_{1}\right)\left(z_{3} \bar{z}_{4}-z_{4} \bar{z}_{3}\right) \sum_{i \neq j=1}^{4}\left(z_{i} \bar{z}_{j}-z_{j} \bar{z}_{i}\right)^{2}}{\left|z_{2}\right|^{2}\left|z_{4}\right|^{2}} d \Omega .
$$

Once again we have a product of a positive sum and a non-vanishing number. We can again show that the SUSY condition (3.3) is not satisfied for Karch-Katz embedding (4.88) without additional magnetic field $F_{2}$. This result is perhaps not so surprising given the fact that Karch-Katz embedding can be regarded as two copies of $w$-embeddings asymptotically, and we have shown that $w$-embedding cannot be supersymmetric unless additional world volume flux is turned on.

\section{Comparison with Benini's proposal}

Benini [41] has proposed a form for the asymptotic flux in the limit of large radius. The purpose of this appendix is to compare our notation with his so that it is clear how our proposal compares with his in the relevant limit.

In the large radius limit, we may take one of the angles $\theta_{1}$ or $\theta_{2}$ to vanish. A helpful piece of intuition is that the worldvolume of the D7-brane in this limit "splits" into two branches, corresponding to one or the other $\theta_{i}$ vanishing.

Let us take the limit where one of the angles, say $\theta_{2}$, is small, and then work order-byorder in $\theta_{2}$. The D7-brane worldvolume in the compact directions can be described by the coordinates $\theta_{1}, \theta_{2}, \phi_{1}, \phi_{2}$. However, to compare with the large radius limit we will trade $\theta_{2}$ and $\phi_{2}$ for $r$ and $\psi$.

In this limit, the $(1,1)$ basis forms $P$ and $Q$ take the form

$$
\begin{aligned}
P & \sim\left[\frac{3}{2} \Omega_{11}+3 \frac{d r}{r} \wedge g^{5}\right]+\cot \frac{\theta_{1}}{2}\left(d \theta_{1} \wedge g^{5}-3 \frac{d r}{r} \wedge \sin \theta_{1} d \phi_{1}\right) \\
Q & \sim-\frac{1}{2} \tan \frac{\theta_{1}}{2}\left(d \theta_{1} \wedge g^{5}-3 \frac{d r}{r} \wedge \sin \theta_{1} d \phi_{1}\right)
\end{aligned}
$$


where $g^{5}=d \psi+\cos \theta_{1} d \phi_{1}+\cos \theta_{2} d \phi_{2}$. The claim in [41] was that the term in square brackets in $P$ was a solution to the inhomogeneous Bianchi identity in the large radius limit. This corresponds to a combination of $P$ and $Q$ with $\alpha=2 k$ and $\beta=2 k \cot ^{2} \frac{\theta_{1}}{2}$, or in other words

$$
\hat{\mathcal{F}}=2 k P+\left(\frac{2 k}{\sin ^{2} \frac{\theta_{1}}{2}}-2 k\right) Q
$$

which we have written in a form to make clear the comparison with formulas such as (4.69).

If one took the large radius limit on the other branch, with $\theta_{1}$ small, the form of $\hat{\mathcal{F}}$ is almost the same, with $\theta_{1}$ and $\phi_{1}$ replaced by $\theta_{2}$ and $\phi_{2}$ everywhere, and multiplication by an overall factor of -1 (due to the antisymmetry of the basis two-form $\omega_{2}$ which appears in the background 2-form potential $B_{2}$.) In our formalism, this sign flip is built into the basis forms $P$ and $Q$ which are antisymmetric under the interchange of indices $1 \leftrightarrow 2$, while the functions $\alpha$ and $\beta$ are symmetric.

Finally, note that our asymptotic form for $\alpha$ in the large radius limit (4.84) in the radial coordinates is

$$
\alpha=-2 k \log \log r+\text { const }+O(1 / \log r)
$$

with a leading $\log \log$ term; this term is effectively constant in the leading $1 / \log r$ approximation, but it does affect the field equations at subleading order (and therefore has to be included), as we argued in section 4.3.4.

Open Access. This article is distributed under the terms of the Creative Commons Attribution Noncommercial License which permits any noncommercial use, distribution, and reproduction in any medium, provided the original author(s) and source are credited.

\section{References}

[1] J.M. Maldacena, The large- $N$ limit of superconformal field theories and supergravity, Adv. Theor. Math. Phys. 2 (1998) 231 [Int. J. Theor. Phys. 38 (1999) 1113] [hep-th/9711200] [SPIRES].

[2] S.S. Gubser, I.R. Klebanov and A.M. Polyakov, Gauge theory correlators from non-critical string theory, Phys. Lett. B 428 (1998) 105 [hep-th/9802109] [SPIRES].

[3] E. Witten, Anti-de Sitter space and holography, Adv. Theor. Math. Phys. 2 (1998) 253 [hep-th/9802150] [SPIRES].

[4] L. Randall and R. Sundrum, A large mass hierarchy from a small extra dimension, Phys. Rev. Lett. 83 (1999) 3370 [hep-ph/9905221] [SPIRES].

[5] L. Randall and R. Sundrum, An alternative to compactification, Phys. Rev. Lett. 83 (1999) 4690 [hep-th/9906064] [SPIRES].

[6] H.L. Verlinde, Holography and compactification, Nucl. Phys. B 580 (2000) 264 [hep-th/9906182] [SPIRES].

[7] K. Dasgupta, G. Rajesh and S. Sethi, $M$ theory, orientifolds and G-flux, JHEP 08 (1999) 023 [hep-th/9908088] [SPIRES]. 
[8] B.R. Greene, K. Schalm and G. Shiu, Warped compactifications in $M$ and F-theory, Nucl. Phys. B 584 (2000) 480 [hep-th/0004103] [SPIRES].

[9] S. B. Giddings, S. Kachru and J. Polchinski, Hierarchies from fluxes in string compactifications, Phys. Rev. D 66 (2002) 106006 [hep-th/0105097] [SPIRES].

[10] C.P. Burgess et al., Warped supersymmetry breaking, JHEP 04 (2008) 053 [hep-th/0610255] [SPIRES].

[11] M.R. Douglas, J. Shelton and G. Torroba, Warping and supersymmetry breaking, arXiv:0704. 4001 [SPIRES].

[12] S. Kachru, L. McAllister and R. Sundrum, Sequestering in string theory, JHEP 10 (2007) 013 [hep-th/0703105] [SPIRES].

[13] O. DeWolfe and S.B. Giddings, Scales and hierarchies in warped compactifications and brane worlds, Phys. Rev. D 67 (2003) 066008 [hep-th/0208123] [SPIRES].

[14] S.B. Giddings and A. Maharana, Dynamics of warped compactifications and the shape of the warped landscape, Phys. Rev. D 73 (2006) 126003 [hep-th/0507158] [SPIRES].

[15] A.R. Frey and A. Maharana, Warped spectroscopy: localization of frozen bulk modes, JHEP 08 (2006) 021 [hep-th/0603233] [SPIRES].

[16] G. Shiu, G. Torroba, B. Underwood and M.R. Douglas, Dynamics of warped flux compactifications, JHEP 06 (2008) 024 [arXiv: 0803.3068] [SPIRES].

[17] M.R. Douglas and G. Torroba, Kinetic terms in warped compactifications, arXiv:0805.3700 [SPIRES].

[18] A.D. Linde, Inflation and string cosmology, eConf C 040802 (2004) L024 [J. Phys. Conf. Ser. 24 (2005) 151] [Prog. Theor. Phys. Suppl. 163 (2006) 295] [hep-th/0503195] [SPIRES].

[19] S.H. Henry Tye, Brane inflation: string theory viewed from the cosmos, hep-th/0610221.

[20] J.M. Cline, String cosmology, hep-th/0612129 [SPIRES].

[21] R. Kallosh, On inflation in string theory, Lect. Notes Phys. 738 (2008) 119 [hep-th/0702059] [SPIRES].

[22] C.P. Burgess, Lectures on cosmic inflation and its potential stringy realizations, PoS P 2GC (2006) 008 [arXiv: 0708. 2865] [SPIRES].

[23] L. McAllister and E. Silverstein, String cosmology: a review, Gen. Rel. Grav. 40 (2008) 565 [arXiv:0710.2951] [SPIRES].

[24] P. Candelas and X.C. de la Ossa, Comments on conifolds, Nucl. Phys. B 342 (1990) 246 [SPIRES].

[25] I.R. Klebanov and M.J. Strassler, Supergravity and a confining gauge theory: duality cascades and $\chi_{S B}$-resolution of naked singularities, JHEP 08 (2000) 052 [hep-th/0007191] [SPIRES].

[26] S. Kachru et al., Towards inflation in string theory, JCAP 10 (2003) 013 [hep-th/0308055] [SPIRES].

[27] D. Baumann et al., On D3-brane potentials in compactifications with fluxes and wrapped D-branes, JHEP 11 (2006) 031 [hep-th/0607050] [SPIRES].

[28] D. Baumann, A. Dymarsky, I.R. Klebanov and L. McAllister, Towards an explicit model of D-brane inflation, JCAP 01 (2008) 024 [arXiv:0706.0360] [SPIRES].

[29] A. Krause and E. Pajer, Chasing brane inflation in string-theory, JCAP 07 (2008) 023 [arXiv:0705.4682] [SPIRES].

[30] O. Aharony, A. Fayyazuddin and J.M. Maldacena, The large- $N$ limit of $N=2,1$ field theories from three-branes in F-theory, JHEP 07 (1998) 013 [hep-th/9806159] [SPIRES]. 
[31] A. Karch and E. Katz, Adding flavor to AdS/CFT, JHEP 06 (2002) 043 [hep-th/0205236] [SPIRES].

[32] S. Kachru, R. Kallosh, A.D. Linde and S.P. Trivedi, De Sitter vacua in string theory, Phys. Rev. D 68 (2003) 046005 [hep-th/0301240] [SPIRES].

[33] S. Kuperstein, Meson spectroscopy from holomorphic probes on the warped deformed conifold, JHEP 03 (2005) 014 [hep-th/0411097] [SPIRES].

[34] P. Ouyang, Holomorphic D7-branes and flavored $N=1$ gauge theories, Nucl. Phys. B 699 (2004) 207 [hep-th/0311084] [SPIRES].

[35] C.P. Herzog, I.R. Klebanov and P. Ouyang, D-branes on the conifold and $N=1$ gauge/gravity dualities, hep-th/0205100 [SPIRES].

[36] C.P. Herzog, I.R. Klebanov and P. Ouyang, Remarks on the warped deformed conifold, hep-th/0108101 [SPIRES].

[37] I.R. Klebanov and A.A. Tseytlin, Gravity duals of supersymmetric $\mathrm{SU}(N) \times \mathrm{SU}(N+M)$ gauge theories, Nucl. Phys. B 578 (2000) 123 [hep-th/0002159] [SPIRES].

[38] M. Mariño, R. Minasian, G.W. Moore and A. Strominger, Nonlinear instantons from supersymmetric p-branes, JHEP 01 (2000) 005 [hep-th/9911206] [SPIRES].

[39] J. Gomis, F. Marchesano and D. Mateos, An open string landscape, JHEP 11 (2005) 021 [hep-th/0506179] [SPIRES].

[40] D.S. Freed and E. Witten, Anomalies in string theory with D-branes, hep-th/9907189 [SPIRES].

[41] F. Benini, A chiral cascade via backreacting D7-branes with flux, JHEP 10 (2008) 051 [arXiv:0710.0374] [SPIRES].

[42] Aavailable at http://www.freefem.org.

[43] D. Arean, D.E. Crooks and A.V. Ramallo, Supersymmetric probes on the conifold, JHEP 11 (2004) 035 [hep-th/0408210] [SPIRES].

[44] M.J. Strassler, The duality cascade, hep-th/0505153 [SPIRES].

[45] I.R. Klebanov and E. Witten, Superconformal field theory on threebranes at a Calabi-Yau singularity, Nucl. Phys. B 536 (1998) 199 [hep-th/9807080] [SPIRES].

[46] N. Seiberg, Electric-magnetic duality in supersymmetric nonAbelian gauge theories, Nucl. Phys. B 435 (1995) 129 [hep-th/9411149] [SPIRES].

[47] F. Benini, F. Canoura, S. Cremonesi, C. Núñez and A.V. Ramallo, Backreacting flavors in the Klebanov-Strassler background, JHEP 09 (2007) 109 [arXiv:0706.1238] [SPIRES].

[48] I. Affleck, M. Dine and N. Seiberg, Dynamical supersymmetry breaking in supersymmetric QCD, Nucl. Phys. B 241 (1984) 493 [SPIRES].

[49] F. Bigazzi, A.L. Cotrone and A. Paredes, Klebanov-Witten theory with massive dynamical flavors, JHEP 09 (2008) 048 [arXiv:0807.0298] [SPIRES].

[50] T.S. Levi and P. Ouyang, Mesons and flavor on the conifold, Phys. Rev. D 76 (2007) 105022 [hep-th/0506021] [SPIRES].

[51] C.P. Burgess, R. Kallosh and F. Quevedo, De Sitter string vacua from supersymmetric D-terms, JHEP 10 (2003) 056 [hep-th/0309187] [SPIRES].

[52] K. Dasgupta, P. Franche, A. Knauf and J. Sully, D-terms on the resolved conifold, JHEP 04 (2009) 027 [arXiv: 0802.0202] [SPIRES]. 